PREPARED FOR THE U.S. DEPARTMENT OF ENERGY, UNDER CONTRACT DE-AC02-76CH03073

PPPL-3783

PPPL-3783

UC-70

3-D Force-balanced Magnetospheric Configurations

by

Sorin Zaharia and C.Z. Cheng

February 2003

N/M

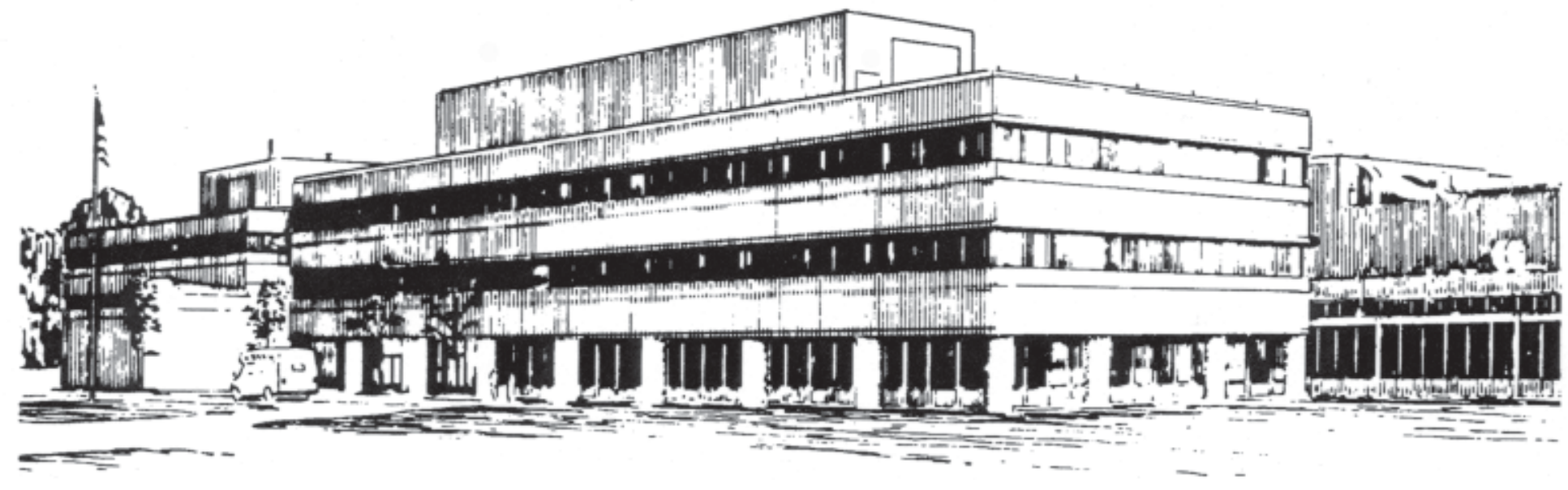

PRINCETON PLASMA PHYSICS LABORATORY PRINCETON UNIVERSITY, PRINCETON, NEW JERSEY 


\section{PPPL Reports Disclaimer}

This report was prepared as an account of work sponsored by an agency of the United States Government. Neither the United States Government nor any agency thereof, nor any of their employees, makes any warranty, express or implied, or assumes any legal liability or responsibility for the accuracy, completeness, or usefulness of any information, apparatus, product, or process disclosed, or represents that its use would not infringe privately owned rights. Reference herein to any specific commercial product, process, or service by trade name, trademark, manufacturer, or otherwise, does not necessarily constitute or imply its endorsement, recommendation, or favoring by the United States Government or any agency thereof. The views and opinions of authors expressed herein do not necessarily state or reflect those of the United States Government or any agency thereof.

\section{Availability}

This report is posted on the U.S. Department of Energy's Princeton Plasma Physics Laboratory Publications and Reports web site in Fiscal Year 2003. The home page for PPPL Reports and Publications is: http://www.pppl.gov/pub_report/

DOE and DOE Contractors can obtain copies of this report from:

U.S. Department of Energy

Office of Scientific and Technical Information

DOE Technical Information Services (DTIS)

P.O. Box 62

Oak Ridge, TN 37831

Telephone: (865) 576-8401

Fax: (865) 576-5728

Email: reports@adonis.osti.gov

This report is available to the general public from:

National Technical Information Service

U.S. Department of Commerce

5285 Port Royal Road

Springfield, VA 22161

Telephone: $1-800-553-6847$ or

(703) $605-6000$

Fax: (703) 321-8547

Internet: http://www.ntis.gov/ordering.htm 


\title{
3-D force-balanced magnetospheric configurations
}

\author{
Sorin Zaharia and C. Z. Cheng \\ Plasma Physics Laboratory, Princeton University, P.O. Box 451, Princeton, NJ 08543, USA \\ K. Maezawa \\ Institute of Space and Astronautical Science, Yoshinodai 3-1-1, Sagamihara, Kanagawa, 229 8510, \\ Japan
}

Abstract. The knowledge of plasma pressure is essential for many physics applications in the magnetosphere, such as computing magnetospheric currents and deriving mag-netosphere-ionosphere coupling. A thorough knowledge of the 3-D pressure distribution has however eluded the community, as most in-situ pressure observations are either in the ionosphere or the equatorial region of the magnetosphere. With the assumption of pressure isotropy there have been attempts to obtain the pressure at different locations by either (a) mapping observed data (e.g. in the ionosphere) along the field lines of an empirical magnetospheric field model, or (b) computing a pressure profile in the equatorial plane (in 2-D) or along the Sun-Earth axis (in 1-D) that is in force balance with the magnetic stresses of an empirical model. However, the pressure distributions obtained through these methods are not in force balance with the empirical magnetic field at all locations. In order to find a global 3-D plasma pressure distribution in force balance with the magnetospheric magnetic field we have developed the MAG-3D code, that solves the 3-D force balance equation $\mathbf{J} \times \mathbf{B}=\nabla P$ computationally. Our calculation is performed in a flux coordinate system in which the magnetic field is expressed in terms of Euler potentials as $\mathbf{B}=\nabla \psi \times \nabla \alpha$. The pressure distribution, $P=P(\psi, \alpha)$, is prescribed in the equatorial plane and is based on satellite measurements. In addition, computational boundary conditions for $\psi$ surfaces are imposed using empirical field models. Our results provide 3-D distributions of magnetic field, plasma pressure as well as parallel and transverse currents for both quiet-time and disturbed magnetospheric conditions.

\section{Introduction}

The magnetospheric plasma pressure is a quantity essential to many physical processes. In particular, plasma pressure regulates the magnetospheric currents, thus strongly affecting the magnetosphereionosphere (M-I) coupling through the change in the field-aligned currents.

Significant effort in space physics research have generally been concentrated on the magnetospheric magnetic field, which has been subject to extensive observations and modeling. Among the magneto- spheric field models developed, probably the most popular are the empirical models, in which one postulates the structure of the magnetospheric currents, and the model parameters are obtained by fitting the model field to an array of observations. Those observations represent data collected by many spacecraft at different locations and at different times, and thus the empirical models describe large-scale time-averaged magnetospheric states rather than instantaneous "snapshots" of the magnetospheric field. Among the better known empirical models we mention those by Olson and Pfitzer [1974]; Ostapenko 
and Maltsev [1997]; Tsyganenko [1987, 1989, 1995, 2002]; Tsyganenko and Stern [1996]. As opposed to the rather extensive observations and studies of the magnetic field, the magnetospheric plasma pressure is much less known. Direct measurements of the pressure over a large domain are scarce, yet the knowledge of the pressure is extremely important from a physical point of view, especially in regions such as the plasma sheet where the plasma parameter $\beta$ (the ratio of plasma pressure to magnetic pressure) has very large values [e.g., Borovsky et al., 1997]. Because in regions of large $\beta$ the pressure critically determines the magnetic field, it is of crucial importance to know the global pressure distribution. One way of obtaining such a global distribution, starting from scarce pressure data, will be presented in this study.

We start our paper by a succinct review of several pressure observations, both at low altitudes (in the ionosphere) and further in the plasma sheet in the magnetospheric tail. We then briefly describe the way these observations have commonly been used in space physics research, mostly in conjunction with empirical magnetic field models. Then, we put forth the theoretical and numerical background of our method of obtaining force-balanced magnetospheric configurations. Finally, we present computational results for both quiet and active-time magnetosphere, with both isotropic and bi-Maxwellian plasma pressure distributions.

\section{Plasma pressure - observations and modeling}

\section{Pressure observations}

In the magnetosphere, the plasma pressure tensor has generally only two distinct diagonal components, corresponding to directions perpendicular and parallel to the ambient magnetic field. Their values can straightforwardly be obtained once the particle distributions are known, being given by (ignoring the flow effects) $P_{\perp}=1 / 2 \int m v^{2} f(\mathbf{v}) \sin ^{2} \beta d^{3} v$ and $P_{\|}=\int m v^{2} f(\mathbf{v}) \cos ^{2} \beta d^{3} v$, respectively, where $f(\mathbf{v})$ is the velocity distribution function of the particle population, while $m$ and $\beta$ represent the particle mass and pitch angle, respectively. When the particle distribution is isotropic, such is the case in the plasma sheet (see below), the plasma pressure can be described by a scalar: $P=P_{\perp}=P_{\|}$.

Quite a few plasma pressure measurements have been performed using observations of particle distribution functions in the plasma sheet, a region which plays a very important role, due to its large $\beta$, in the dynamics of the magnetosphere. Both in situ observations [Stiles et al., 1978; Nakamura et al., 1991] and theoretical studies [Noetzel et al., 1985; Hill and Voigt 1992] overwhelmingly suggest that the pressure in the plasma sheet is isotropic. Among plasma sheet pressure measurements at distances $|X|>10 \mathrm{R}_{\mathrm{E}}$, we mention observations using ISEE spacecraft: ISEE1 [Huang and Frank, 1994] and ISEE2 [Spence et al., 1989; Angelopoulos et al., 1993]. Closer to Earth, where plasma pressure is generally bi-Maxwellian, we mention AMPTE/CCE [Lui and Hamilton, 1992; De Michelis et al., 1999] and AMPTE/IRM [Angelopoulos et al., 1993] measurements. Finally, probably the most extensive observations of plasma sheet pressure are those of the GEOTAIL mission [e.g., Hori et al., 2000]. Besides in situ plasma sheet measurements, a novel technique [Wing and Newell, 1998] has allowed imaging of plasma sheet ions by analyzing their precipitation at low altitudes in the ionosphere. The theoretical background of the method relies on the isotropization of plasma sheet ions when the ratio of their gyroradius to the magnetic field curvature exceeds a certain value [Sergeev et al., 1993]. Observing the distribution of field-aligned precipitating ions in the ionosphere one thus obtains an accurate reflection of their isotropic distribution function in the plasma sheet. Based on the theory of Sergeev et al. [1993], Wing and Newell [1998] have inferred the plasma sheet pressure contribution due to protons by observing particle precipitation in the ionosphere at latitudes higher than the so-called "isotropy boundary." Their method relies on the technique, commonly employed in the space physics community, of relating the 2-D pressure measurements to different points in space (thus obtaining a 3-D distribution) by "mapping" the pressure using empirical magnetic field models. The implicit assumptions in such an approach are: i). the plasma pressure is assumed to be isotropic; and ii). the observed pressure is considered to be in force balance with the magnetic stresses of the empirical model field.

\section{Plasma pressure and empirical field models; lack of force balance}

Another series of approaches actually goes further than using empirical model fields for mapping observed plasma pressure distributions and try to infer the pressure from the empirical model itself, by attempting to calculate the pressure values that would be in force balance with the model fields in 1-D along 
the Sun-Earth axis [e.g., Spence et al., 1987; Lui et al., 1994] or in 2-D in the equatorial plane [Horton et al., 1993; Cao and Lee, 1994]. One important question poses itself with regard to this approach, and the question is "Can the empirical model fields be equilibrated by plasma pressure of a certain pressure tensor form ?" Partially answering the question, Zaharia and Cheng [2002] have shown that the field of the T96 empirical model cannot be balanced globally with isotropic plasma pressure. Their findings show that while in 1-D along the Sun-Earth axis the Lorentz force $|\mathbf{J} \times \mathbf{B}|$ is a good approximation to the observed values of $|\nabla P|$, in more than 1-D the quiet-time T96 field cannot be in equilibrium with an isotropic pressure. Other studies have shown that anisotropic pressure profiles in equilibrium with empirical magnetic fields can only be found on a maximum of two planes [Horton et al., 1993; Cao and Lee, 1994], as the problem is over-determined in 3-D. With the lack of global force balance, the use of empirical field models in conjunction with observed pressure profiles can give rise to physical errors [Zaharia and Cheng, 2002].

\section{Possible solutions to the force balance problem}

As pointed out by Stern [1994], the most probable reason for the lack of equilibrium in the empirical field models is the loss of accuracy in the derivatives of the model magnetic field $\mathbf{B}$ - while the model field can provide a good approximation to the observed field through least-square fitting, the comparison between the derivatives of the model and observed field, respectively, will not present such a good correlation.

An alternate method is then needed to obtain force-balanced magnetospheric states. One such method that computes a $3-\mathrm{D}$ magnetospheric equilibrium is the "ballistic" frictional approach [Hesse and Birn, 1992, 1993; Toffoletto et al., 2001], in which an empirical magnetic field structure is used as an initial state in a modified-MHD simulation. The approach usually seeks to find force-balanced states in which the magnetic field is not very different from the initial empirical field [Toffoletto et al., 2001]. The final force-balanced magnetospheric state in the frictional method is not unique however, depending on the choice of the polytropic index $\gamma$ [Hesse and Birn, 1993].

While the "ballistic" frictional method obtains 3D force-balanced states in a large spatial domain, it does not reproduce the very large $\beta$ values commonly observed in the plasma sheet, especially dur- ing disturbed magnetospheric times. Such $\beta$ values are however reproduced by the method presented in this paper, which consists in numerically solving the 3 -D equilibrium equation in a flux coordinate system. In the computation we use observation-based pressure profiles and realistic boundary conditions as input. The results we present in this paper are obtained using plasma pressure in the plasma sheet from GEOTAIL observations [e.g., Hori et al., 2000], or along the midnight meridian in the equatorial plane, from the so-called Spence-Kivelson formula [Spence and Kivelson, 1993], as well as anisotropic pressure closer to Earth obtained from AMPTE/CCE measurements [Lui, 1993; Lui et al., 1994; De Michelis et al., 1999]. The computation is performed inside a domain defined by magnetic flux boundaries obtained from Tsyganenko's T96 and T01 empirical field models [Tsyganenko, 1995; Tsyganenko and Stern, 1996; Tsyganenko, 2002].

\section{Calculation of 3-D quasi-equilibrium}

In the rationalized EMU unit system, the equilibrium with isotropic pressure $P$ can be expressed as

$$
\mathbf{J} \times \mathbf{B}=\nabla P
$$

It is generally believed that such an equilibrium exists in the "slow-flow" region of the magnetosphere (the inner and middle magnetosphere and on closedfield lines) at all times [Wolf, 1983], except during periods of very explosive magnetospheric activity such as substorm onset and expansion phases.

\section{Euler potential representation of B; flux coordinate system}

From $\nabla \cdot \mathbf{B}=0$, the vector $\mathbf{B}$ can be expressed as

$$
\mathbf{B}=\nabla \psi \times \nabla \alpha,
$$

where $\psi$ and $\alpha$ are called Euler potentials [e.g., Stern, 1967]. Obviously $\mathbf{B} \cdot \nabla \psi=\mathbf{B} \cdot \nabla \alpha=0$ and thus the intersection of constant $\psi$ and $\alpha$ surfaces defines the magnetic field lines. Our 3-D equilibrium computation will be made in a flux coordinate system in which two of the coordinates are chosen to be the Euler potentials defining the magnetic field. There is freedom however in the choice of the third coordinate, $\chi$, representing the position along the field line. A particular choice for this coordinate is equivalent to choosing a specific form for the Jacobian of the $(\psi, \alpha, \chi)$ system, $\mathcal{J}=[(\boldsymbol{\nabla} \psi \times \boldsymbol{\nabla} \zeta) \cdot \boldsymbol{\nabla} \chi]^{-1}[$ Cheng 
et al., 1993; Cheng, 1995]. Our choice for $\chi$ in this study will be the "equal arc length" [Cheng, 1995; Zaharia and Cheng, 2002], such that equal $d \chi$ variations correspond to equal length variations $d s$ along a field line.

\section{Equilibrium equations in flux coordinate system}

In the flux coordinate system $\{\psi, \alpha, \chi\}$, by considering the components of the equilibrium equation, Eq. (1), in the directions of $(\mathbf{B} \times \boldsymbol{\nabla} \psi)$ and $(\mathbf{B} \times \boldsymbol{\nabla} \alpha)$, respectively, one has:

$$
\begin{gathered}
\mathbf{J} \cdot \boldsymbol{\nabla} \psi=\boldsymbol{\nabla} \cdot\left[(\boldsymbol{\nabla} \psi)^{2} \boldsymbol{\nabla} \alpha-(\boldsymbol{\nabla} \alpha \cdot \boldsymbol{\nabla} \psi) \boldsymbol{\nabla} \psi\right]=-\frac{\partial P}{\partial \alpha}, \\
\mathbf{J} \cdot \boldsymbol{\nabla} \alpha=\boldsymbol{\nabla} \cdot\left[(\boldsymbol{\nabla} \alpha \cdot \boldsymbol{\nabla} \psi) \boldsymbol{\nabla} \alpha-(\boldsymbol{\nabla} \alpha)^{2} \boldsymbol{\nabla} \psi\right]=\frac{\partial P}{\partial \psi},
\end{gathered}
$$

as obtained before [e.g., Birn et al., 1977; Cheng, 1995; Zaharia and Cheng, 2002]. In the work presented here, the equations (3) and (4) are solved numerically using an improved version of the MAG-3D code [Cheng, 1995], by considering observation-based pressure distributions $P(\psi, \alpha)$ and realistic boundary conditions for $\psi$ obtained from empirical field models.

The two coupled equations above are second-order inhomogeneous elliptic partial differential equations (PDEs) for $\alpha$ and $\psi$ in the $(\alpha, \chi)$ and $(\psi, \chi)$ coordinate spaces, respectively, and they admit unique solutions if boundary conditions and the inhomogeneous terms on the right-hand sides (RHS) are prescribed. Besides the boundary conditions, one also needs to specify the inhomogeneous terms in equations (3) and (4), i.e. $\partial P / \partial \psi$ and $\partial P / \partial \alpha$. While Cheng [1995] used analytical profiles $P=P(\psi)$ to calculate near-Earth magnetospheric configurations, such a choice is not too realistic for the plasma sheet farther than about $10 R_{E}$ from Earth [Zaharia and Cheng, 2002]. In this paper we present results with $P(R, \phi, Z=0)$ from observations as input $(R, \phi$ and $Z$ in this paper define the usual cylindrical coordinate system, with the Earth in the center). The $P(R, \phi, Z=0)$ functional is kept fixed, i.e. we are interested in finding a magnetic field configuration in equilibrium with given 2-D pressure profile in the equatorial plane. To do this, the function $P(\psi, \alpha)$ will be changed at each iteration as $\psi$ and $\alpha$ change, in order to maintain the input pressure profile fixed in space.

\section{Field-aligned currents}

The field-aligned currents can be calculated numerically once the magnetic field is known, from Ampere's law: $J_{\|}=(\boldsymbol{\nabla} \times \mathbf{B}) \times \mathbf{B} / B$. This method however presents accuracy problems due to the large values of the magnetic field close to the Earth's surface. A more accurate computation of the field-aligned currents can be performed by noting that in a quasiequilibrium state with isotropic $P$, the component of the electric current parallel to the magnetic field, $J_{\|}$, can be obtained from the charge neutrality condition $\boldsymbol{\nabla} \cdot \mathbf{J}=0$ by the so-called Vasyliunas relation [ Vasyliunas, 1970, 1984]:

$$
\mathbf{B} \cdot \boldsymbol{\nabla}\left(\frac{J_{\|}}{B}\right)=\frac{\nabla B^{2} \times \mathbf{B} \cdot \nabla P}{B^{4}}=\frac{2 \mathbf{B} \cdot(\nabla P \times \kappa)}{B^{2}} .
$$

An integration of Eq. (5) along the field line readily provides $J_{\|}$if the quasi-equilibrium magnetic field configuration is known. The calculation of $J_{\|}$using Vasyliunas relation was performed before by Cheng [1995], in a computed 3-D magnetospheric state in force balance with a $P(\psi)$ profile. The reverse problem was considered by Antonova and Ganyushkina [1996], who obtained $\nabla P$ at the ionosphere by using observed $J_{\|}$there [Iijima and Potemra, 1976] and empirical magnetic fields.

\section{Numerical method; boundary conditions and pressure input}

\section{Computational domain}

Our computational domain is a topologically closed region, delimited by the inner and outer magnetic flux surfaces, $\psi_{\text {in }}$ and $\psi_{\text {out }}$, which are kept fixed throughout the computation. In this work the $\psi_{\text {in }}$ and $\psi_{\text {out }}$ surfaces are obtained by field-line mapping using the latest empirical models, T96 and T01. The advantage of using these models is the possibility of quantifying the level of activity of the magnetosphere, by a proper choice of the solar wind parameters: $P_{\mathrm{SW}}$ (solar wind dynamic pressure), $B_{\mathrm{IMF}}$ (interplanetary magnetic field) and DST index - that enter as input in the models. We note again that even though the inner and outer flux boundaries are being kept fixed during the computation, the magnetic field lines are never fixed, even on those surfaces, as they have the ability to move along the surfaces throughout the iterative process. 
Ideally, one would start the magnetic field line mapping from the Earth's surface, such that the computational domain would be closed by a band representing the surface of revolution of the arcs of circle of the Earth's surface between the lower and higher latitudes corresponding to $\psi_{\text {in }}$ and $\psi_{\text {out }}$, respectively. The inner and outer flux surfaces delimiting a typical closed domain can be seen in Fig. 1.

While this choice of starting points can be considered in some cases (when the outer flux boundary considered is not very far from Earth), generally if one wants to include the mid- and far-tail regions of the plasma sheet then computational problems may appear with such a choice. Specifically, if the domain extends too far in the magnetotail, the discrepancy between the very strong magnetic field at the Earth's surface and the weak field in the tail, coupled with the strong deformation of the flux coordinate system in real space leads to huge values and steep gradients in the Jacobian $\mathcal{J}$ [e.g., Becker et al., 2001], giving rise to numerical problems in the computation. Therefore, in those cases we do not extend the domain down to the Earth's surface, but instead only down to a sphere of radius $r>1 R_{E}$ enveloping the Earth but close enough to it that the deviations of the field on the surface of the sphere from a dipole field are not significant.

\section{Inverse representation; iterative computational method}

The two coupled equilibrium equations (3) and (4) are solved numerically in a computational flux coordinate system $(\rho, \zeta, \theta)$ using an inverse iterative technique [Cheng, 1995]. We consider a numerical grid in this system, made of $N_{\psi} \times N_{\alpha} \times N_{\chi}$ computational grid points. When the equilibrium is reached, the Euler potentials $\psi$ and $\alpha$ defining the magnetic field are only functions of $\rho$ and $\zeta$, respectively. In our approach, the magnetic field configuration is expressed in "inverse form", i.e. we compute $\mathbf{r}(\rho, \zeta, \theta)$. In order to have a large grid density in regions of interest, the computational points are generally not equidistant in real space. Instead, $\rho$ is chosen such that equal $\rho$ intervals correspond to equal $d r$ intervals in real space along the midnight axis in the equatorial plane; $\zeta$ is chosen such that a large grid point density is concentrated in the vicinity of midnight local time (the area of largest plasma $\beta$ ). The third computational flux coordinate is obtained from the equal arc length choice: $\theta=\chi$.

In the above computational flux coordinate sys- tem, the boundary conditions that need to be specified for the 2 equations, (3) and (4), are values of $\alpha$ and $\psi$ on the boundaries of the $(\zeta, \theta)$ and $(\rho, \theta)$ domains, respectively. Specifically, for Eq. (3) for $\alpha$, the boundary conditions in the $\zeta$ coordinate are simply prescribed by periodicity. In the $\theta$ coordinate, the boundary conditions for $\alpha$ are obtained from the knowledge of the values of $\alpha$ at the ends of each field line. This knowledge comes from the fact that the magnetic field at the Earth's surface can be assumed to be overwhelmingly due to internal Earth sources [Tsyganenko, 1990]. By only taking into account the highly-dominant dipole term on the Earth's surface, we simply have $\alpha \equiv \phi$ on the Earth's surface [e.g., Stern, 1970]. This boundary condition is approximately correct even when we do not extend the domain down to the Earth's surface, but only to a sphere of radius $r$ enveloping the Earth, with $r$ not too large. Now for Eq. (4) for $\psi$, again boundary conditions in the $\theta$ coordinate means knowing the value of $\psi$ at both ends of a field line. If those are on the Earth surface and again consider the field there to be dipolar, their value of $\psi$ is then analytically known [e.g., Stern, 1967; Cheng, 1992, 1995]: $\psi=-B_{D} \mathrm{R}_{\mathrm{E}}^{2} \sin ^{2} \Theta$, where $B_{D}$ and $\mathrm{R}_{\mathrm{E}}$ are the equatorial dipole field on the Earth's surface and the Earth radius, respectively, while $\Theta$ represents the colatitude. Finally, the boundary condition for $\psi$ in the $\rho$ coordinate is obtained from empirical field models by mapping magnetic field lines with footpoints on the Earth's surface corresponding to the inner and outer flux surfaces, $\psi_{\text {in }}$ and $\psi_{\text {out }}$.

A configuration $\mathbf{r}(\rho, \zeta, \theta)$ is needed as the starting point in the iterative procedure, and usually we take this to be the configuration given by the empirical model. The inverse iterative technique consists in the following steps: (1) on the constant $\psi=\psi(\rho)$ surfaces, Eq. (3) is solved for $\alpha$ in the $(\zeta, \theta)$ space, with the inhomogeneous term $\partial P / \partial \psi$ kept constant; (2) the result of the previous calculation is a function $\alpha(\rho, \zeta, \theta)$; based on this solution, new $\alpha=\alpha[\zeta(x, y, z)]$ $=$ constant surfaces are obtained by moving the grid points in $(\zeta, \theta)$ space on each $\psi$ surface using cubic spline interpolation; (3) on each $\alpha(\zeta)=$ constant surface, Eq. (4) is solved in $(\rho, \theta)$ space, keeping the inhomogeneous term $\partial P / \partial \alpha$ fixed; (4) new $\psi[\rho(x, y, z)]$ $=$ constant surfaces are obtained by moving the grid points in $(\rho, \theta)$ space on each constant $\alpha$ surface. A procedure typically needed in iterative equilibrium calculations to ensure numerical stability is the socalled "blending" [e.g., Hudson et al., 2002], a pro- 


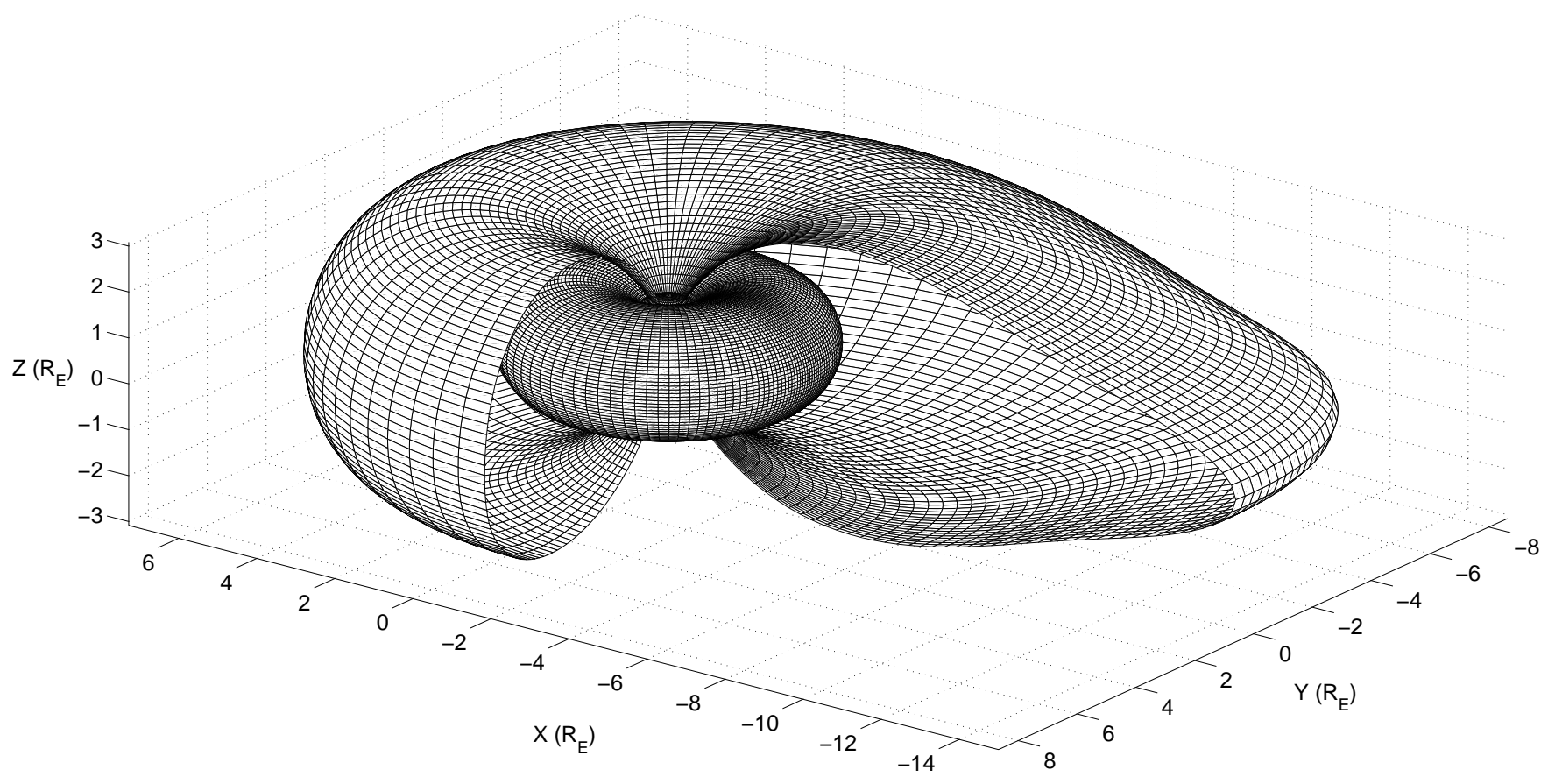

Figure 1. Inner and outer flux $(\psi)$ surfaces enveloping the computational domain (obtained here by field line mapping of quiet-time T01 model). The inner surface, seen through a cut in the outer flux surface, is almost a torus, due to the quasi-axisymmetry in magnetic field so close to Earth's (the Earth, not visible in the figure, is at $[\mathrm{X}, \mathrm{Y}, \mathrm{Z}]=[0,0,0])$. The outer surface however is highly asymmetric, due to magnetic field stretching in the magnetotail. Constant $\chi$ lines and $\alpha$ lines (the latter also representing the $\mathbf{B}$ field lines) are also shown on both flux surfaces.

cess through which some fraction of the solution of the previous iteration is "blended" into the latest iterative solution: $\psi^{(n+1)} \leftarrow \gamma_{\psi} \psi^{(n)}+\left(1-\gamma_{\psi}\right) \psi^{(n+1)}$; $\alpha^{(n+1)} \leftarrow \gamma_{\alpha} \alpha^{(n)}+\left(1-\gamma_{\alpha}\right) \alpha^{(n+1)}$, where $\gamma_{\psi}$ and $\gamma_{\alpha}$ are the blending parameters.

The iterative steps described, with blending parameters chosen empirically for maximum convergence rate, are repeated until the $\alpha=$ constant and $\psi=$ constant surfaces converge to some tolerance. One simple criterion for convergence is represented by the cumulative difference between the values for $\alpha$ or $\psi$ between two consecutive iterations $n$ and $n-1$ (e.g. for $\alpha$ : $\Sigma^{(\alpha n)}=\Sigma_{i, j, k}\left|\alpha_{i, j, k}^{(n)}-\alpha_{i, j, k}^{(n-1)}\right|$ ). A more physical measure of the convergence is the value of the force "imbalance" at iteration $n$, defined in a manner similar to [Toffoletto et al., 2001] as $\|F\|=\int|\mathbf{J} \times \mathbf{B}-\nabla P| d V / \int d V$. We normalize this force imbalance to the initial imbalance, $\left\|F_{0}\right\|$, by considering the quantity $f_{n}=\left\|F_{n}\right\| /\left\|F_{0}\right\|$ and following its decrease throughout the iterations.

\section{Results with different pressure profiles}

In this section we present several computed quietand active-time 3-D magnetospheric quasi-equilibria, in which the magnetic fields are in force balance with different observed pressure distributions: the so-called Spence-Kivelson formula [Spence and Kivelson, 1993], plasma sheet pressure from the GEOTAIL satellite, as well as anisotropic pressure profiles observed near Earth by AMPTE/CCE [Lui and Hamilton, 1992; De Michelis et al., 1999]. In all cases presented in this paper, we take the tilt of the Earth's magnetic axis to be zero for simplicity.

\section{Quiet-time computation with $P$ input from Spence-Kivelson empirical formula}

The Spence-Kivelson empirical formula (hereinafter, the SK formula) was obtained for quiet-time conditions along the midnight meridian in the equatorial plane by Spence and Kivelson [1993], who employed 
non-linear least-square fitting of quiet-time pressure data, mainly from ISEE-2 observations. The explicit form of the SK formula is $P(\mathrm{nPa})=89 e^{-0.59|X|}+$ $8.9|X|^{-1.53}$. It has been shown [Zaharia and Cheng, 2002 ] that the pressure values given by the SK formula are very close to values obtained by integrating the $\mathbf{J} \times \mathbf{B}$ force of the quiet-time T96 empirical model along the equatorial midnight meridional line. Since the SK formula is only valid along that line, additional assumptions have to be made with regard to the azimuthal (local-time) dependence of the equatorial plane pressure before we can employ the formula in our 3-D code. In this paper we present results with equatorial $P(R, \phi, Z=0)=P(R, Z=0)$, where $R$ is the distance from Earth in the equatorial plane. This should not be an unreasonable choice, since observations [e.g., Wing and Newell, 1998] report the pressure further than about $7 R_{E}$ to vary little in the azimuthal $(\phi)$ direction. Closer to Earth on the other hand, the plasma $\beta$ generally tends to be quite low, and therefore the pressure does not influence much the magnetic field configuration. It has to be mentioned that even with this pressure choice, the computed magnetospheric state is still non-axisymmetric, due to the lack of axisymmetry (tail stretching) in the boundary conditions. Other code runs, not presented in this paper, with a small azimuthal $(\phi)$ dependence lead to to magnetic field configurations very similar to the $P(R, \phi, Z=0)=P(R, Z=0)$ case presented here, with the only significant differences in the fieldaligned current configurations.

In order to portray a quiet-time magnetosphere, the $\psi_{\text {in }}$ and $\psi_{\text {out }}$ flux boundaries of our domain are obtained by field mapping using the T96 model, parameterized by $D S T=-5 \mathrm{nT}, P_{\mathrm{SW}}=2.1 \mathrm{nPa}$, $B_{\mathrm{yIMF}}=0$ and $B_{\mathrm{zIMF}}=1 \mathrm{nT}$, representing average solar wind parameters during quiet times as obtained from the OMNI solar wind database. The computational domain is limited by a sphere of radius $r=4 R_{E}$ enveloping the Earth, in order to avoid numerical problems, as described before. The latitudes chosen for tracing the inner and outer flux surfaces are such that the field lines mapped using the model at midnight eventually intersect the equatorial plane at $X=-5 R_{E}$ and $X=-23 R_{E}$, respectively. The initial field configuration considered in the computation is the one given by the T96 model with the solar wind parameters specified above. This configuration is not in equilibrium with an isotropic pressure profile [Zaharia and Cheng, 2002].

The final force-balanced state is achieved by the
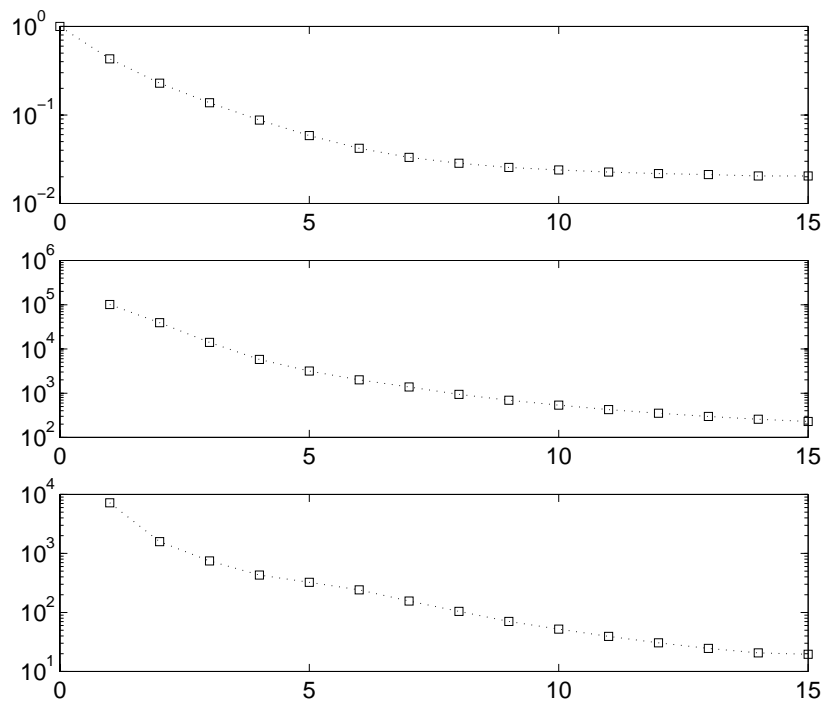

Figure 2. (a) The decrease with iteration number $n$ of the following quantities in the SK case: normalized force imbalance $f_{n}$ (top); cumulative $\psi$ change between consecutive iterations (middle); and cumulative $\alpha$ change between consecutive iterations (bottom).

code after typically less than 20 iterations. Figure 2 shows the decrease in the normalized force imbalance $f_{n}$ defined before vs. the iteration number $n$, as well as decreases in the $\psi$ and $\alpha$ "errors" previously defined. The decrease with $n$ is monotonic for all three quantities, and is quite steep during the first iterations. The force imbalance in the final state is down to about $1 / 50$ of its initial value (which is normalized to be 1). Most of the decrease takes place during the first 10 iterations, after which the force imbalance almost reaches a plateau. With further iterations, the force imbalance does not decrease much, however the computational "errors" for $\psi$ and $\alpha$ continue to decrease at a significant rate. This can be due to the non-linear nature of the computation, whereby the plateau reached by $f_{n}$ approaches the minimum value of force imbalance reachable in the finite-difference computation with the number of grid points considered. Our number of grid points, $N=201 \times 65 \times 101$, seems however to be more than adequate for accurately computing a force-balanced state, as seen in the significant decrease of the the force imbalance $f_{n}$ [c.f. Toffoletto et al., 2001]. Inspection of several physical parameters of the magnetospheric state throughout the iterations reinforces this conclusion, with the convergence actually setting in after iteration $n=7$, as the physical parameters do not change more than $5 \%$ 

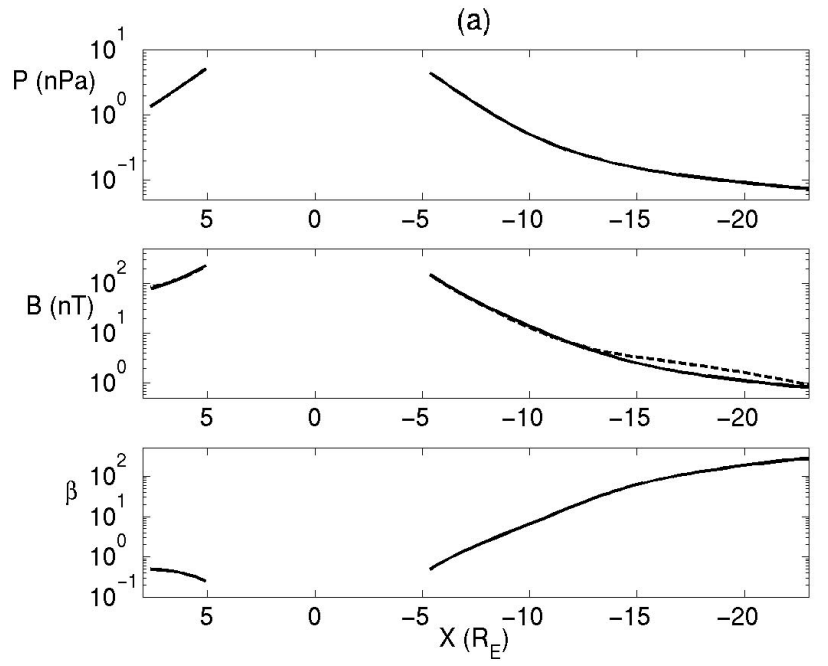

(b)

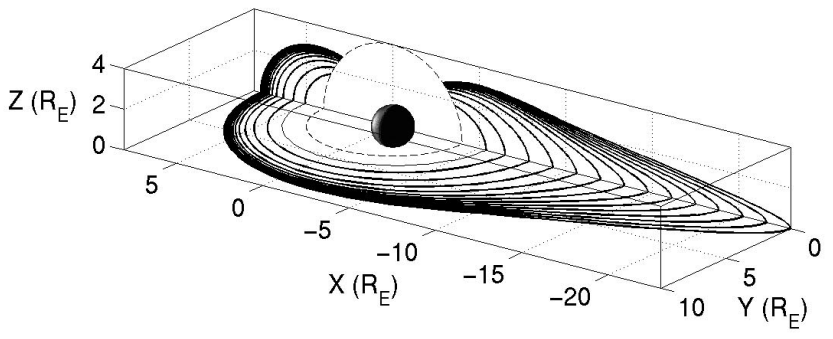

Figure 3. (a) Profiles along the Sun-Earth axis for $P,|\mathbf{B}|$ and $\beta$ in the quiet-time 3-D equilibrium with SpenceKivelson pressure; the dashed line in the second plot represents $|\mathbf{B}|$ from the T96 model; (b) Noon-midnight and equatorial plane cross-sections of constant flux surfaces in the north-western quadrant for the computed 3$\mathrm{D}$ equilibrium; dashed lines show semi-circles of radius $4 R_{E}$, corresponding to the sphere at the inner domain boundary.

(a)

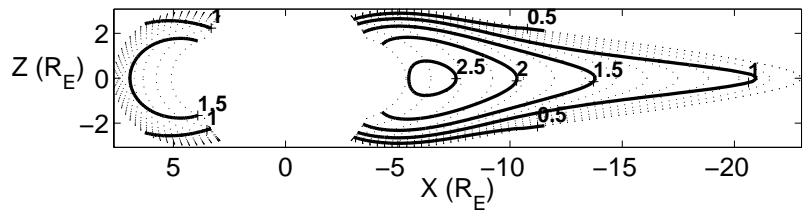

(b)

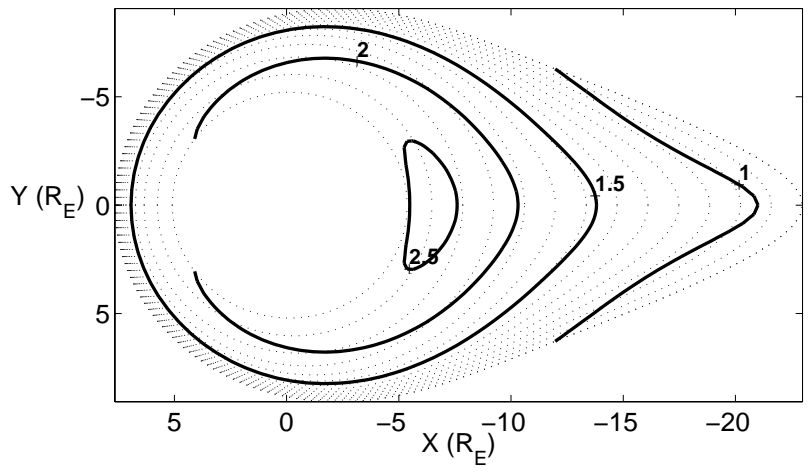

(c)

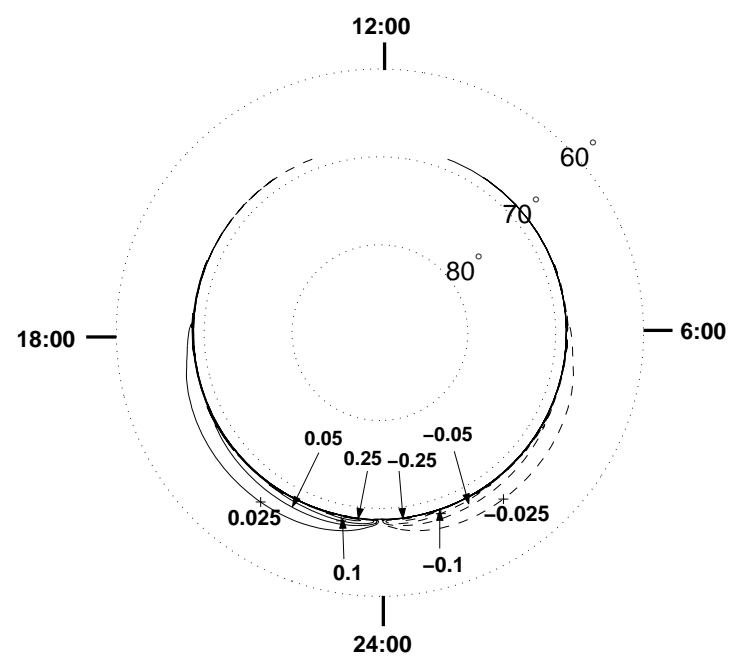

Figure 4. (a) Contours of constant transverse current $J_{\perp}$ in the noon-midnight and equatorial planes for the quiettime 3-D equilibrium with Spence-Kivelson pressure; the dotted lines represent constant $\psi$ contours; (b) Contours of field-aligned current $J_{\|}$in the ionosphere (at $1 R_{E}$, computed by assuming $J_{\|} / B=$ const. between 1 and $4 R_{E}$ ). 
(a)
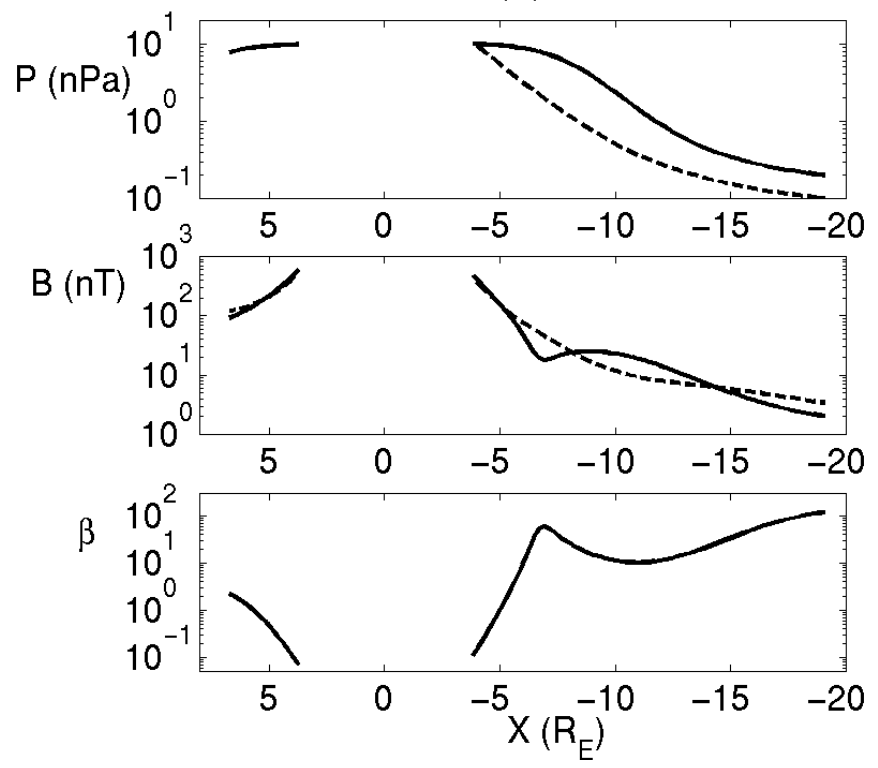

(b)
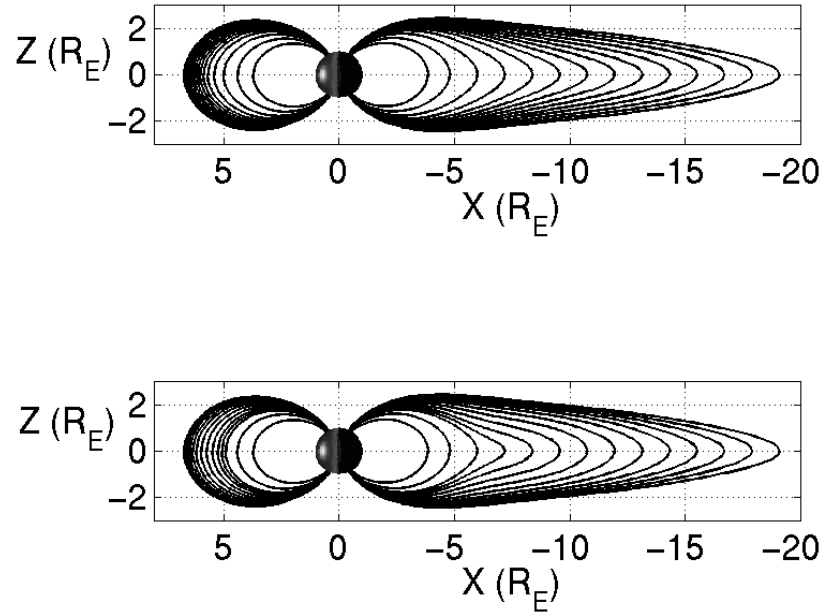

Figure 5. (a) Sun-Earth axis profiles of $P,|\mathbf{B}|$ and $\beta$ for the disturbed-time computation; the dashed line in the top plot represents the SK pressure used in the previous case, while the dashed lines in the middle plot show $|\mathbf{B}|$ from the T96 model; (b) Noon-midnight plane cross-sections of constant flux surfaces for the Tsyganenko field and the computed 3-D disturbed-time equilibrium field, respectively.

between $n=7$ and the final state at $n=15$.

Several physical quantities along the Sun-Earth axis characterizing the computed equilibrium state are presented in plot (a) of Fig. 3: the pressure $P$ (basically the SK formula), together with the magnetic field magnitude $B$ and plasma $\beta$. One observes that $B$ decreases while $\beta$ increases monotonically with $|X|$ on the night side. The very large values of $\beta$ are only attained for $|X|>20 R_{E}$, with a maximum of around 150 at $23 R_{E}$. In the $B$ plot we also show by dashed lines the value of the magnetic field of the T96 model, seen to be close to the computed value. This fact is not surprising, considering that the SK pressure profile agrees very well [Zaharia and Cheng, 2002] with the integral of $\mathbf{J} \times \mathbf{B}$ along the equatorial midnight meridian in quiet-time T96.

Plot (b) of Fig. 3 shows the north-western quadrant of the closed field-line magnetospheric domain under consideration, with constant $\psi$ contours of the computed equilibrium state plotted in the noon-midnight and equatorial planes. The electric currents of the computed 3-D equilibrium are presented in Fig. 4. Plots (a) and (b) in the figure show contours of constant transverse current $J_{\perp}\left(\mathbf{J}_{\perp} \cdot \mathbf{B}=0\right)$ in the noonmidnight and equatorial planes, respectively. The current reaches a maximum of $2.6 \mathrm{nA} / \mathrm{m}^{2}$ at around $7 R_{E}$, its spatial location is rather broad in the $Z$ direction and extends to all local times. The contours also show that the ring current gradually transforms into cross-tail current at larger distances from Earth, without a definite boundary between the two current systems. Plot (c) of Fig. 4 shows the fieldaligned currents (FACs) as computed from Vasyliunas relation, Eq. (5) in the obtained force-balanced state. The FACs are mostly of region- 2 sense, with a maximum current density of around $0.25 \mu \mathrm{A} / \mathrm{m}^{2}$. The total Region-2 sense current is found by integration to be 2.8 MA. While this latter value is rather typical for a quiet-time magnetosphere, the current density is a bit on the low side. This is most probably an artifact of the $P(R, Z=0)$ pressure dependence considered. Other code runs where a $\phi$-dependence was introduced in the pressure profile lead to a significant increase in the field-aligned current. The Region- 1 current is almost non-existent, its total value amounting to only $0.09 \mathrm{MA}$. The fact that there are no significant Region-1 currents (which again, might be changed by introducing a $\phi$ dependence in $P$ ) does not mean that they could not be present in the plasma sheet at $20 R_{E}$ away from midnight for example, just that they are 
not included in our computational domain.

\section{Disturbed-time computation}

In order to obtain a quasi-equilibrium state characteristic of a disturbed magnetospheric time the pressure profile used in the quiet-time case (the SK formula) is modified such that pressure values close to Earth (between 5 and $8 R_{E}$ ) are increased, consistent with observations [e.g., Lui et al., 1987; Kistler et al., 1992] showing larger plasma pressure and Earthward pressure gradients during periods of magnetospheric activity. The modification, which models both of the above observed features, is to consider the equatorial $P$ of the form $P(R, Z=0)=P_{0}\left[1+\tanh \left(X_{1}-\right.\right.$ $\left.R) / \Delta_{1}\right]+P_{S K}\left[1+\tanh \left(R-X_{2}\right) / \Delta_{2}\right]$, where $P_{S K}$ is the corresponding pressure profile at distance $R$, while $P_{0}$, $x_{1}, x_{2}, \Delta_{1}, \Delta_{2}$ are constants. In this paper we choose $P_{0}=5 \mathrm{nPa}, X_{1}=8, X_{2}=9.25$, and $\Delta_{1}=\Delta_{2}=2.5$. The pressure profile, shown at the top of Plot (a) in Fig. 5, reaches a value of $2 P_{S K}$ tailward at distances significantly farther than $9 R_{E}$, twice the corresponding quiet-time pressure at the same distances. The pressure values are however more than twice their quiet-time value between 5 and $10 R_{E}$, consistent with observations. The inner and outer flux surfaces for this case are again obtained by field-line mapping using the T96 empirical field model, however with different parameters: $D S T=-40 \mathrm{nT}, P_{\mathrm{SW}}=5.0 \mathrm{nPa}$, $B_{\mathrm{yIMF}}=0$ and $B_{\mathrm{zIMF}}=-2 \mathrm{nT}$, typical for active magnetospheric times.

Besides pressure $P$, the left plot of Fig. 5 presents Sun-Earth axis profiles of other physical parameters in the computed magnetospheric equilibrium. The value of the equilibrium magnetic field $B$ is seen to first decrease monotonically with increasing $|X|$ at midnight, however $B$ soon reaches a local minimum of about $15 \mathrm{nT}$ at a distance of $7 R_{E}$. The dip in the $B$-field value also corresponds to a peak in plasma $\beta$, with $\beta \simeq 100$ around $7 R_{E}$. Finally, looking at Plot (b) of the same figure, we remark a striking difference between the computed flux surfaces (bottom picture) vs. the flux surfaces in the T96 model (top picture) (since at midnight $\alpha=\phi$, the constant $\psi$ contours portrayed in plot (b) in Fig. 5 also represent the field lines). Specifically, the field line curvature in the computed magnetospheric state in the region between 7 and $10 R_{E}$ on the nightside is much larger than the curvature of the T96 field lines in the same region.

The large curvature suggests a strong current sheet in that region, which can indeed be seen in Plot (a) of Fig. 6, which shows the noon-midnight meridional plane contours of constant $J_{\perp}$. The maximum current value, of about $15 \mathrm{nA} / \mathrm{m}^{2}$ at $7 R_{E}$, is almost an order of magnitude larger than the corresponding quiettime picture. The current sheet has a limited extent in $Z$ and also in azimuth, as can be seen in Plot (b) of the same figure. The existence of such current sheets has been observationally confirmed during disturbed magnetospheric times, such as a substorm growth phase. Understanding their appearance and features is crucial to hot research topics, from substorm onset mechanisms to space weather. A more detailed study of the disturbed-time current sheets is relegated to a future paper. Plot (c) of the figure shows the fieldaligned currents in this case, which are predominantly of Region-1 sense. The maximum value of the current density is $2.6 \mu \mathrm{A} / \mathrm{m}^{2}$, typical of values measured in the ionosphere for disturbed times. The total current values for the Region-1 and Region-2 currents are 1.18 MA and 0.27 MA, respectively. Again, an azimuthal dependence in the pressure distribution could change the $J_{\|}$results.

\section{Pressure input from GEOTAIL}

While in the previous two cases presented the $\alpha$ dependence of $P(\psi, \alpha)$ was dictated by the choice $P=P(R, Z=0)$, in this section we present results with a more realistic $2-D$ pressure input in the equatorial plane from GEOTAIL satellite data. The GEOTAIL data represent spatial and temporal averages of plasma pressure (considered isotropic) at different points in a 2-D spatial domain in the nightside, delimited by the GEOTAIL apogee of about $30 R_{E}$ and perigee of $10 R_{E}$. The data at each point is the average in space of 1-minute time-averaged pressure observations in a rectangle of dimensions $1 \mathrm{R}_{\mathrm{E}}$ in the $X$ direction and $2 \mathrm{R}_{\mathrm{E}}$ in the $Y$ direction, centered around that point. Further, the GEOTAIL data is sorted according to magnetospheric activity as a function of $K_{p}$. In this section we present results obtained using low-activity data, defined by $0<K_{p}<1$. This pressure data is presented in Plot (a) of Fig. 7.

Before using the GEOTAIL data as input into the code, a certain degree of smoothing is necessary. Because of very high $\beta$ values in the plasma sheet (for example, the plasma $\beta$ as measured by GEOTAIL can exceed 100), even slight variations in $P$ can lead to dramatic changes in the magnetic field configuration in force balance with the pressure. The raw GEOTAIL data is generally characterized by sharp gradients, which cannot be equilibrated by any equilibrium magnetic field configuration. The very sharp gradi- 
(a)

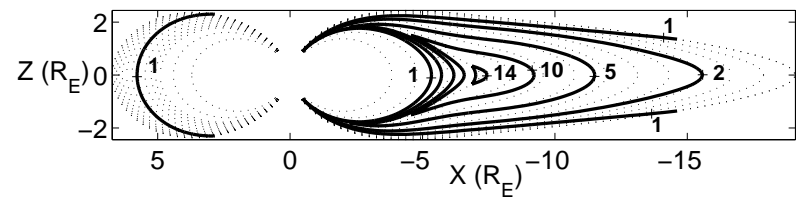

(b)

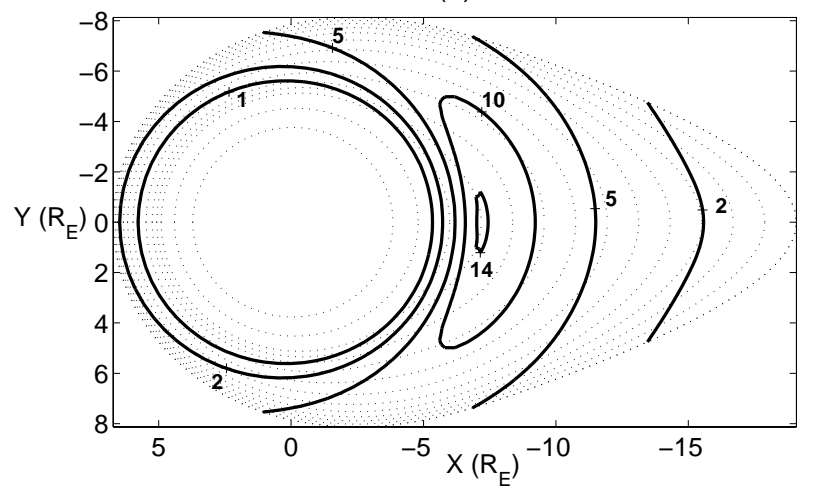

(c)

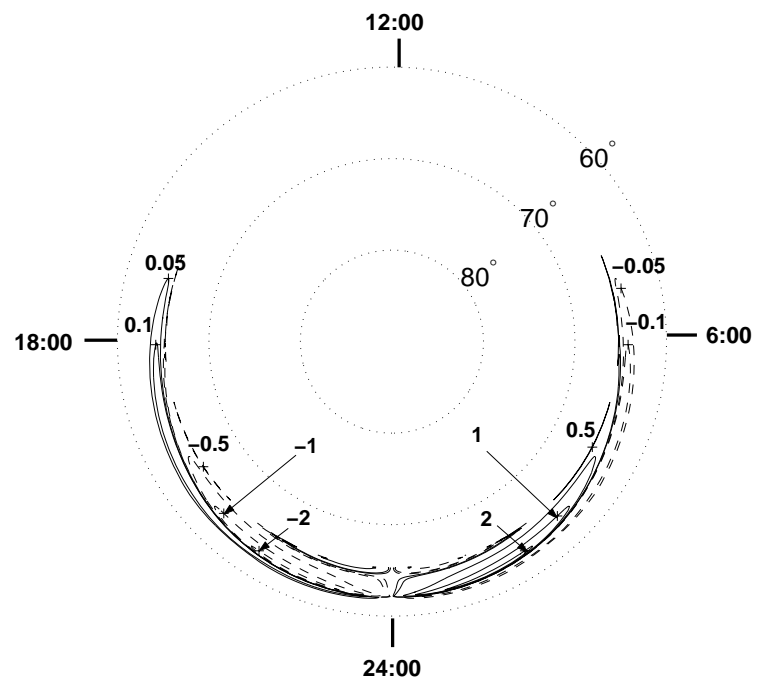

Figure 6. (a) Contours of constant transverse current $J_{\perp}$ in the noon-midnight and equatorial planes for the disturbed-time equilibrium; the dotted lines represent constant $\psi$ contours; (b) Contours of field-aligned current $J_{\|}$in the ionosphere (at $1 R_{E}$ in our model).

ents do not necessarily have a physical meaning, their existence being more likely due to the limited amount of data, which might be insufficient for completely averaging out the time variations (note that the data for any two adjacent cells among those shown in Fig. 7 may come from different orbits of the satellite, separated time-wise by more than five days). A solution to the problem is applying standard image processing techniques to the raw pressure data, however such a method is not very objective - while sharp gradients will indeed be smoothed, no physical measure exists as to what degree of smoothing is necessary such that vital information about the spatial dependence of the pressure is not lost. For the above reason, we employed a more phenomenological approach, by employing a least-square fit of the GEOTAIL data against a chosen 2-D function. We only considered the data in the spatial domain delimited by $-20<X<$ $-8 R_{E}$ and $-10<Y<10 R_{E}$ (this restrained spatial domain was chosen for reasons of numerical convergence). There were 183 rectangles of $1 R_{E} \times 2 R_{E}$ size containing data points in this domain. The fitting function was taken in the form $P(R, \phi, Z=0)=$
$\left(A+B \mathrm{e}^{-C R}+D R^{E}\right) \cdot \sum_{m} F_{m} \sin (m \phi)$. The fit is thus non-linear in $R$ and $\phi$, and has 14 parameters: $A, B, C, D, E$ and the different $F_{m}$, with $0 \leq m \leq 8$. The angle $\phi$ was defined as $\phi=\arcsin (Y / R)$, such that $\phi$ has opposite signs in the eastern vs. the western hemisphere, and the series above is able to reproduce the observed dawn-dusk asymmetry in the pressure. The resulting fit is quite accurate, as described by its correlation coefficient $\mathcal{R}=0.92$. While only nightside GEOTAIL pressure was used for the least-square fit, we nevertheless employ the obtained $P(R, \phi, Z=0)$ function on the dayside as well in our 3 -D equilibrium code. While of course there are no guarantees about the accuracy of the formula when applied to dayside, the low dayside plasma $\beta$ (generally $<1$ ) makes any pressure inaccuracies unable to change much the magnetic field configuration there.

Plot (b) of Fig. 7 shows the profiles along the SunEarth axis for pressure, magnetic field and plasma $\beta$ in the computed equilibrium with the pressure fitted vs. the GEOTAIL data taken as input (the inner and outer flux surfaces were obtained by T96 field-line mapping, with the same T96 parameters as in the SK 
(a)

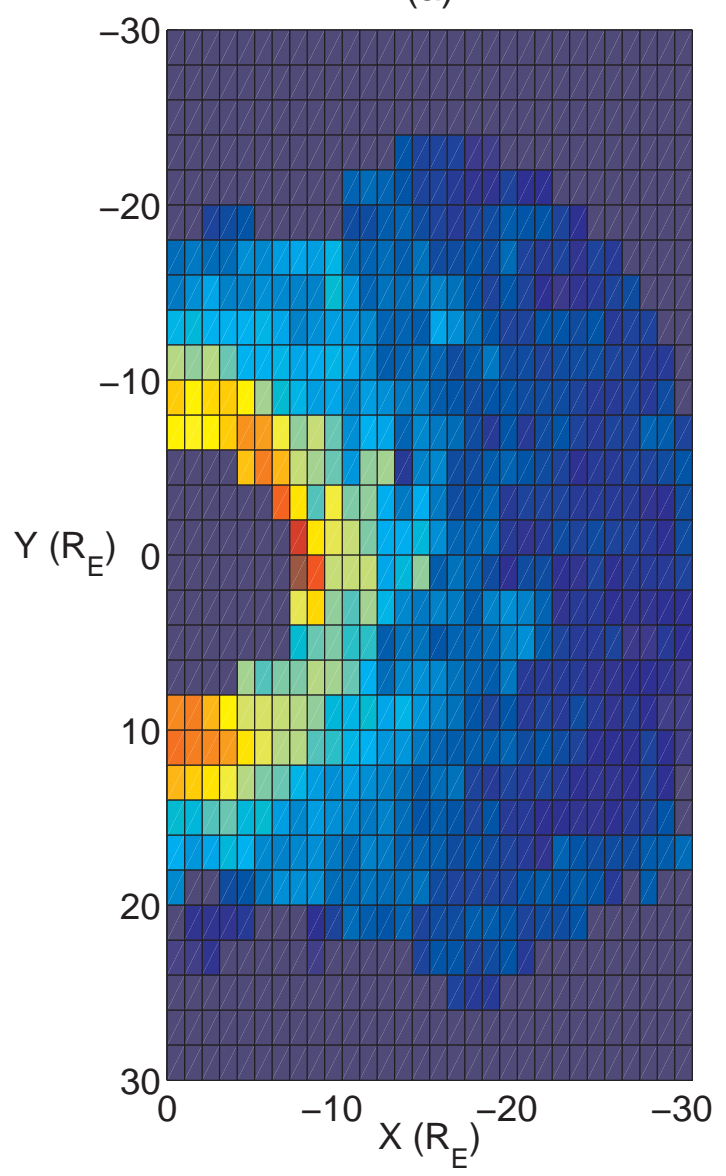

$\mathrm{P}(\mathrm{nPa})$

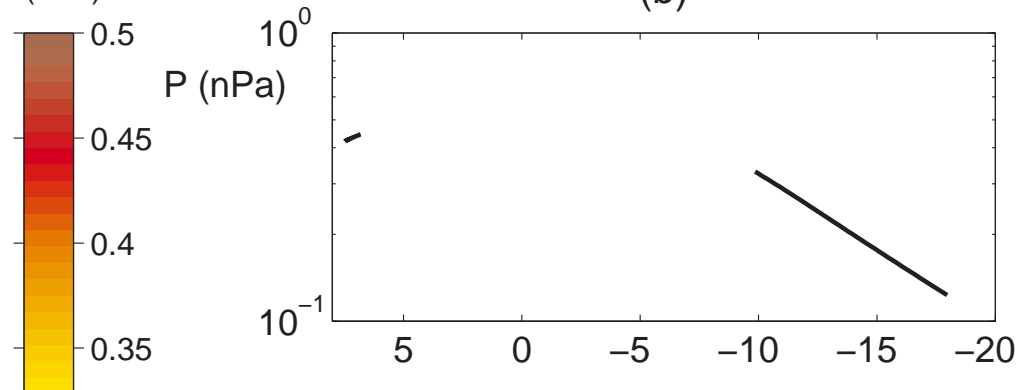

$-0.3$

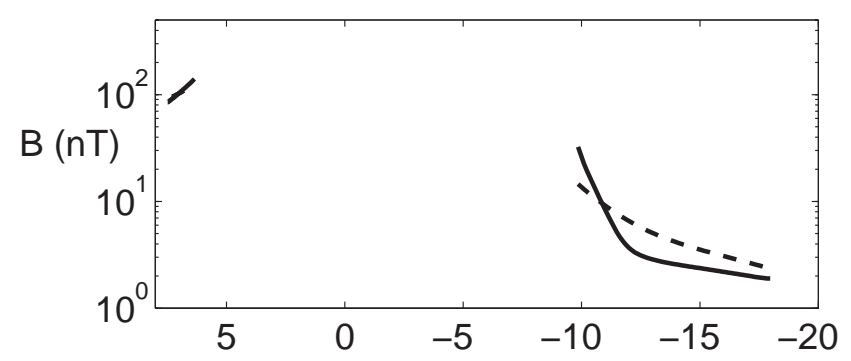

0.15

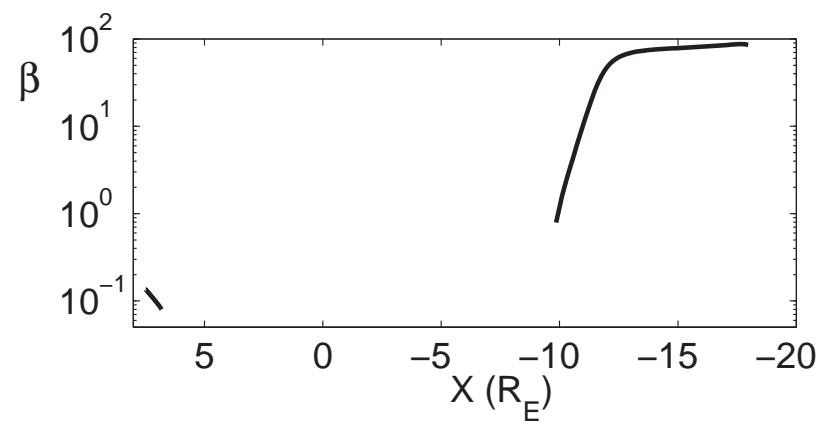

Figure 7. (a) GEOTAIL plasma sheet pressure data for times of low magnetospheric activity $\left(0<K_{p}<1\right)$; (b) Sun-Earth axis profiles of $P,|\mathbf{B}|$ and $\beta$ for the computation with GEOTAIL plasma sheet pressure; the dashed line in the second plot represents $|\mathbf{B}|$ from the T96 model.

case; due to considerations of numerical stability, the outer surface was chosen to extend only to $-18 R_{E}$ in the nightside however). It is seen that the magnetic field of the computed equilibrium is lower in the nightside beyond $11 R_{E}$ than the corresponding T96 field, showing that the field lines of T96 might not be stretched enough to equilibrate observed plasma pressure gradients. Due to the smaller $B$, the plasma $\beta$ is larger compared to the SK case.

The electric current densities, both transverse and parallel, are shown in Fig. 8. While there is no pressure information from GEOTAIL outside the plasma sheet (in this case, for $|X|<8 R_{E}$ )) and therefore there is no ring current in the figure, one can see however that the contours of the cross-tail current are closed on the Earth side, suggesting that in this case, as opposed to the SK case, the ring and crosstail currents are distinct. As opposed to the previous two cases, in which both pressure and boundary conditions have an east-west symmetry, in this case the computed transverse current exhibits a dawn-dusk asymmetry, with the current density actually larger on the dawn side (the maximum value being at around 1:00 local time). This feature is related to the particular dawn-dusk asymmetry (values higher on the dawn side than on the dusk side at $R \approx 12 R_{E}$ in Plot (a) of Fig. 7) in the Geotail pressure data, and that asymmetry is presently not well understood. Since the plasma sheet is typically observed to be thinner on the dusk side, it may be that some of the lower pressure values in the evening sector in the data do not actually belong to the plasma sheet. This would war- 
(a)

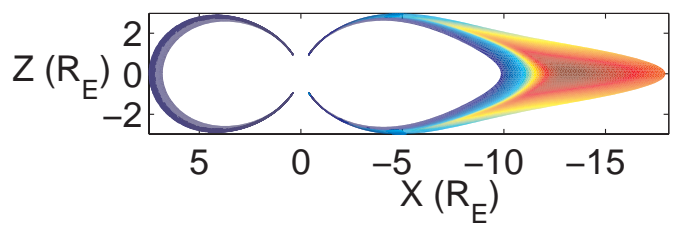

(b)

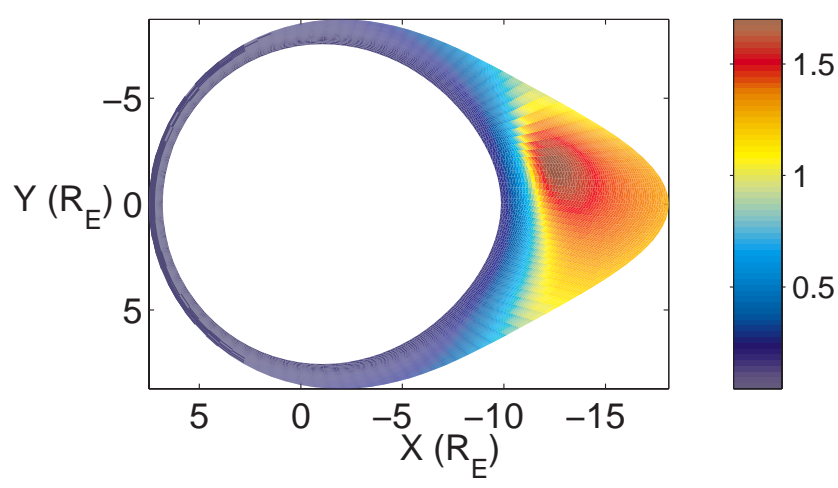

$\mathrm{nA} / \mathrm{m}^{2}$

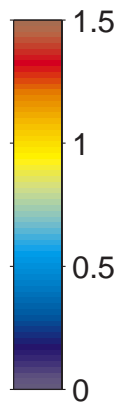

0.5

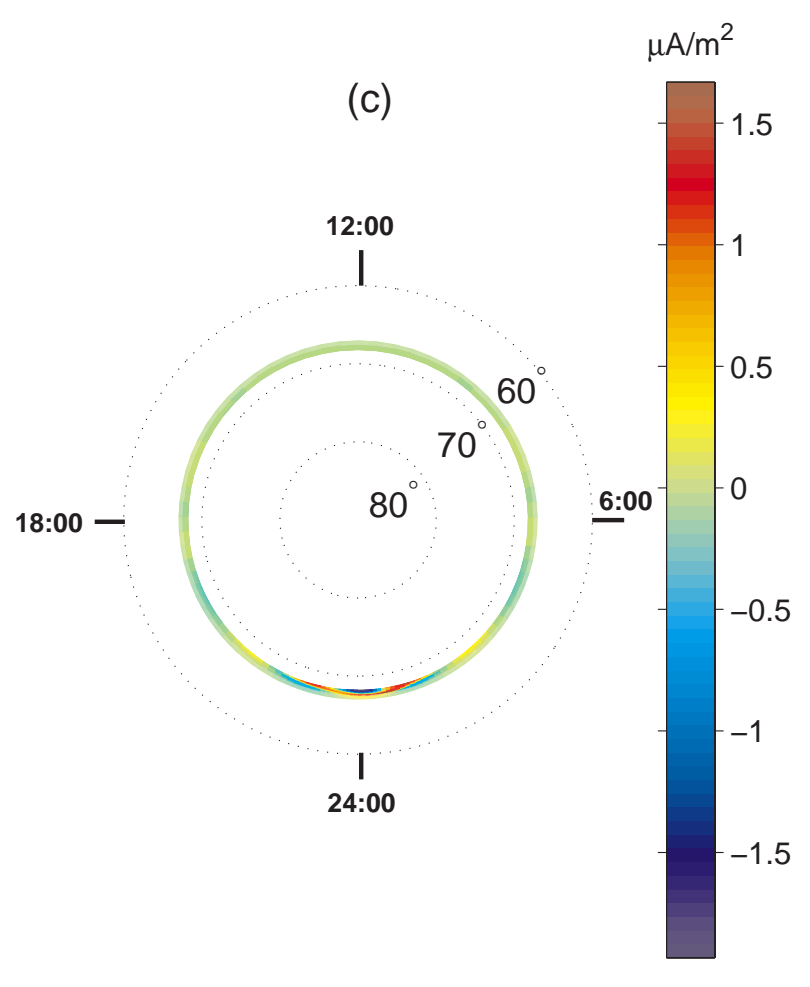

Figure 8. (a) Transverse current $J_{\perp}$ in the noon-midnight and equatorial planes for the equilibrium obtained with GEOTAIL pressure input; (b) Field-aligned current $J_{\|}$in the ionosphere (at $1 R_{E}$ in our model) for the equilibrium with GEOTAIL pressure.

rant in the future both a refinement in the pressure data values considered for the computation, as well as a relaxation of the dawn-dusk symmetry in the computational boundary conditions in our model. Plot (c) of Fig. 8 depicts the field-aligned currents. While our computational domain in this case corresponds to a narrow region in the polar plot, the FACs in this case are less structured than before, due to the more complex pressure input. The FACs, which reach maxima of around $1.93 \mu \mathrm{A} / \mathrm{m}^{2}$ (typical for quiet-time field-aligned current values), are of both Region-1 and Region-2 sense, and overlap in intricate ways. While the current densities are significantly higher than in the SK case (probably due to the azimuthal dependence of the pressure $P$ ), the total Region- 1 and Region-2 currents are only 0.2 and $0.23 \mathrm{MA}$, respectively, due to the limited extent of the computational domain considered in this case.

\section{Anisotropic pressure input from AMPTE/CCE}

The 3 cases presented so far consider the pressure to be isotropic, i.e. described by a scalar $P$. However, at distances close to Earth (generally for $r<15 R_{E}$ ) the pressure has generally been found to be not isotropic but bi-Maxwellian, with the degree of anisotropy $P_{\perp} / P_{\|}$exceeding 2 very close to Earth [Lui and Hamilton, 1992]. For a more accurate description of the near-Earth magnetosphere it is important therefore to obtain a magnetic field structure in force balance with observed anisotropic pressure distributions. The extension of our approach to include anisotropic pressure is straightforward and will not be presented here. The only modifications in the equilibrium equations 3 and 4 appear on the right-hand side for the inhomogeneous terms. In the computation for this case, we use bi-Maxwellian pressure profiles and degrees of anisotropy based on AMPTE/CCE observations [Lui and Hamilton, 1992; De Michelis 
(b)

(a)

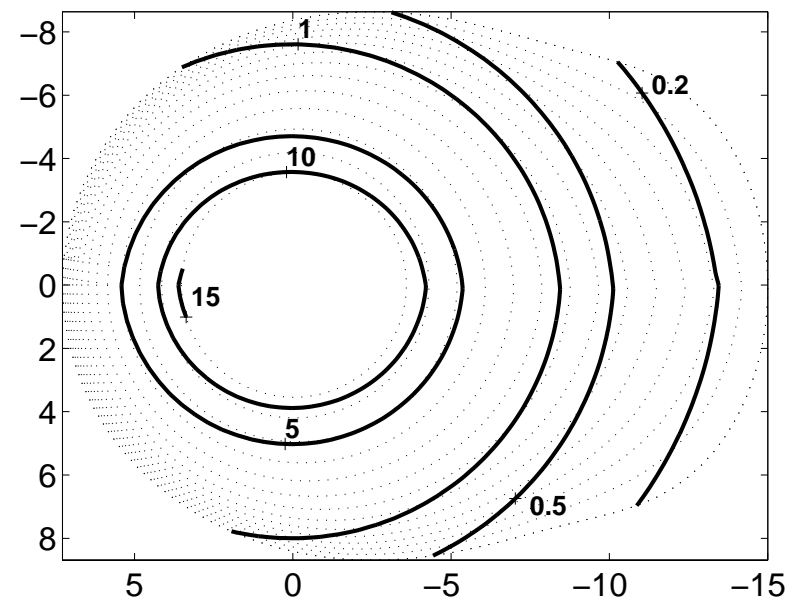

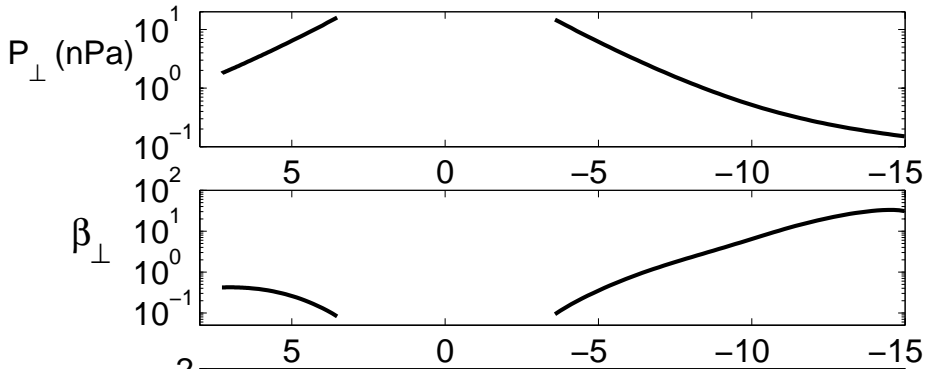

A

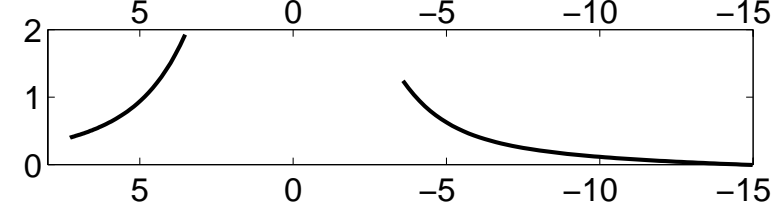

B (nT)

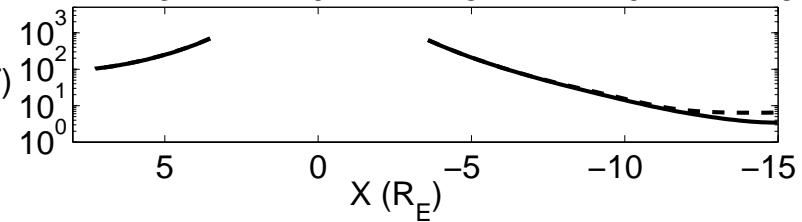

Figure 9. (a) Contours of constant $P_{\perp}$ in the equatorial plane; (b) Profiles along the Sun-Earth axis for: perpendicular pressure $P_{\perp}$, perpendicular $\beta_{\perp}$, degree of anisotropy $A=P_{\perp} / P_{\|}-1$, and magnetic field magnitude $B$.

et al., 1999]. The 2-D profiles obtained by De Michelis et al. [1999] exhibit as a main feature an azimuthal asymmetry in pressure, with higher values at midnight and dusk than at dawn, and the highest pressure at noon local time. On the other hand, the degree of anisotropy is lowest at midnight and higher at other local times, but again with a maximum at noon. For this computation we use the polynomial empirical formula obtained by Lui et al. [1994] for the degree of anisotropy on the midnight meridional line from AMPTE/CCE observations, and we add a simple dependence of the form $2+\cos \phi$ to model its azimuthal variation, in accordance with the qualitative behavior found by De Michelis et al. [1999]. With regard to the pressure values, the "average" pressure (defined as $\left.\langle P\rangle=\left(2 P_{\perp}+P_{\|}\right) / 3\right)$ obtained from AMPTE/CCE on the nightside [Lui and Hamilton, 1992] has been shown [Zaharia and Cheng, 2002] to be very close to the isotropic $P$ values of the SK formula. Therefore, on the equatorial plane we obtain $P_{\perp}$ and $P_{\|}$from the system of equations $P_{\perp}(R) / P_{\|}(R)=A(R, \phi=0)$ and $2 P_{\perp}+P_{\|}=3 P_{S K}(R)$. Finally, we also assume a $\phi$ dependence in $\langle P\rangle$, also of the form $1+a \sin \phi$, with $a$ chosen to model the qualitative azimuthal dependence of pressure describe above. In order to model the dawn-dusk asymmetry we choose $a=-0.2$ in the western hemisphere and $a=0.33$ in the eastern hemisphere. Plot (a) of Fig. 9 shows contours in the equatorial plane of constant perpendicular pressure, $P_{\perp}$. Once $P_{\perp}$ and $P_{\|}$are known in the equatorial plane, their values along the field lines are uniquely determined from energy and magnetic moment conservation [e.g., Cheng, 1992].

The $\psi_{\text {in }}$ and $\psi_{\text {out }}$ surfaces delimiting our computational domain are obtained in this case using the T01 empirical field model [Tsyganenko, 2002]. The advantage of the T01 model vs. T96 is that the former has much better data coverage of the near-Earth region we investigate here. Since the T01 model is only valid for $X>-15 R_{E}$, we choose the outer flux surface $\psi_{\text {out }}$ to only extend to $15 R_{E}$ on the nightside. The parameters we choose in the T01 model are the same as in our previous quiet-time case: $D S T=-5 \mathrm{nT}$, $P_{\mathrm{SW}}=2.1 \mathrm{nPa}, B_{\mathrm{yIMF}}=0$ and $B_{\mathrm{zIMF}}=1 \mathrm{nT}$. Plot (b) of Fig. 9 shows profiles along the Sun-Earth axis of several quantities describing the computed 3-D equilibrium: perpendicular plasma pressure and $\beta, P_{\perp}$ and $\beta_{\perp}$, as well as degree of anisotropy (defined here as $\left.A=P_{\perp} / P_{\|}-1\right)$ and the magnetic field magnitude $B$.

The electric currents obtained in the computed 3-D equilibrium in this case are presented in Fig. 10. The 
(a)

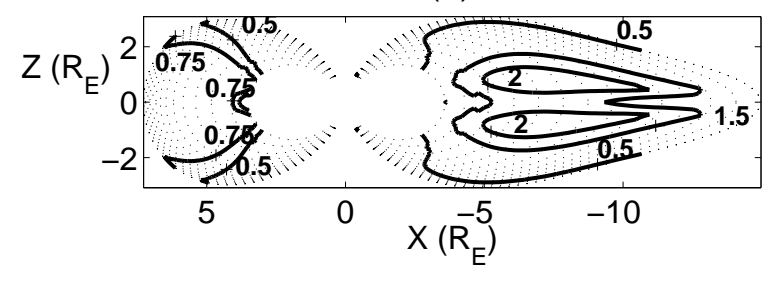

(b)

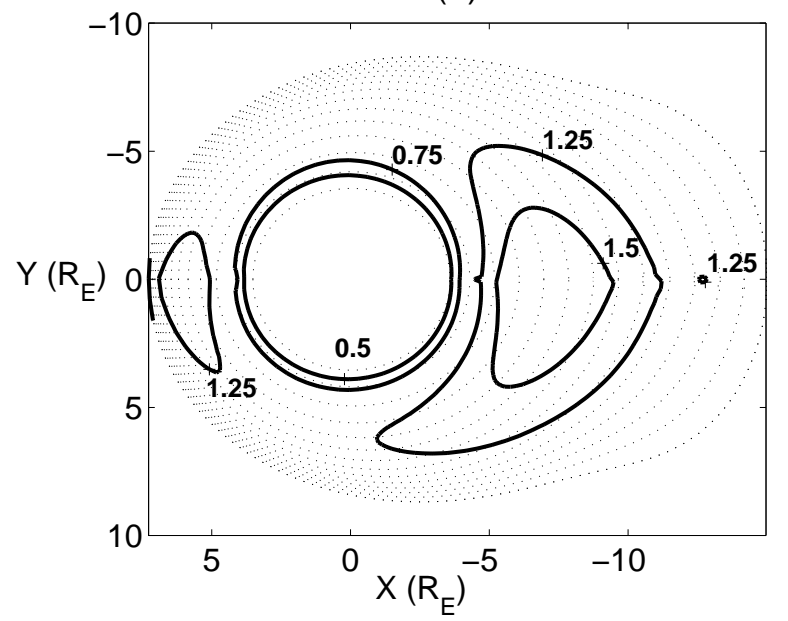

(c)

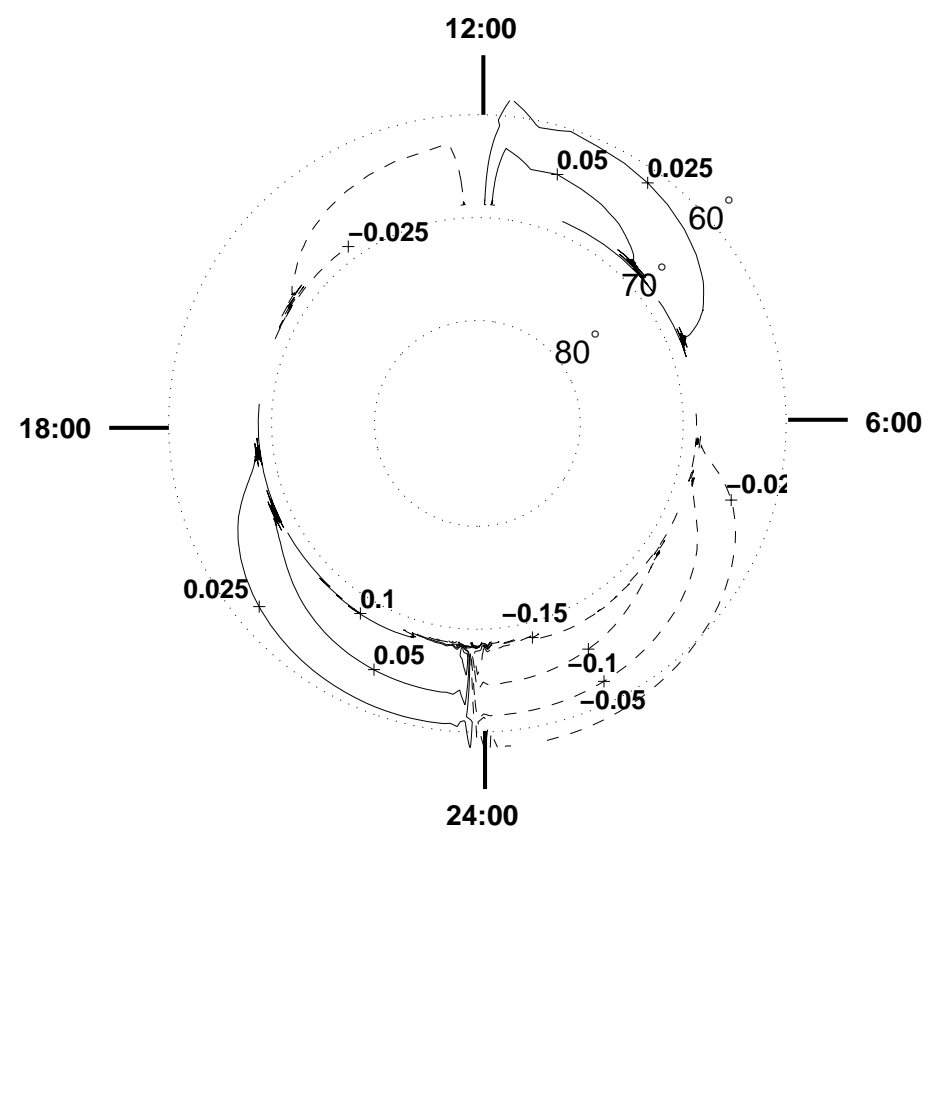

Figure 10. (a) Perpendicular current $J_{\perp}$ contours in the noon-midnight and equatorial planes for a 3-D equilibrium with anisotropic pressure; the dotted lines represent constant $\psi$ contours; (b) Contours of constant $J_{\|}$in the ionosphere (at $\left.1 R_{E}\right)$.

most interesting feature appears in Plot (a) of the figure, showing the fact that the azimuthal (toroidal) current is peaked away from the equator, a result previously obtained for the 2-D case by Cheng [1992]. The maximum transverse current appears in twin regions, above and below the plane, and thus the current is maximum in this case in regions which do not have the maximum field curvature. The field-aligned currents, shown in Plot (c) of Fig. 10, are mostly of Region-2 sense in the nightside, and of Region-1 sense on the dayside. This is consistent with a quiet-time and a domain limited to $|X|<15 \mathrm{R}_{\mathrm{E}}$. The maximum value of the current density is $0.1 \mathrm{nA} / \mathrm{m}^{2}$, however the currents exist in a very large region, spanning many degrees of latitude. This makes the total value of the field-aligned currents to quite significant, at 1.33 MA and 1.15 MA for the Region-1 and Region-2 currents, respectively.

\section{Summary and conclusions}

In addition to knowing the magnetic field vector, the knowledge of the 3-D magnetospheric plasma pressure distribution is needed for many physical applications. There are however no global synoptic observations for the pressure, with most measurements being limited either to low altitudes in ionosphere or to the plasma sheet region. While using the scarce pressure observations in conjunction with empirical field models has been a popular practice in the community, the lack of force balance in those models [Zaharia and Cheng, 2002] poses problems for some 
applications. Consequently, it is imperative to obtain 3-D force-balanced states in which the pressure distribution is in equilibrium with the Lorentz force $\mathbf{J} \times \mathbf{B}$. Using the pressure observations as input for calculating the state would effectively yield 3-D global pressure distributions once the force-balance state is found.

A method to find force-balanced magnetospheric configurations is presented, and it consists in solving the 3-D force-balance equation numerically with computational boundary conditions obtained from empirical field models (T96 or T01) and observation-based pressure profiles as input. We present results for both quiet- and active-time magnetospheres, with a choice of either isotropic or anisotropic (bi-Maxwellian) pressure distributions. All pressure distributions used are based on observations. The level of magnetospheric activity is parameterized by the values of the solar wind parameters in the empirical models determining the boundary conditions, as well as by changes in pressure distributions used as input. The resulting 3 -D force-balanced states successfully reproduce the large observed values of the plasma $\beta$ in the plasma sheet. The knowledge of the force-balanced states is even more important in regions of high plasma $\beta$, as at those locations small variations in pressure can lead to very large changes in the magnetic field configuration.

When comparing the obtained states with the empirical magnetic field structures from the Tsyganenko models, we note that while during quiet times the force-balanced configuration is not too different from the field predicted by the empirical model, for disturbed magnetospheres this is not true anymore, as the equilibrium configuration can be radically different from the empirical Tsyganenko field. For the disturbed time with isotropic pressure presented, we find that a thin cross-tail current sheet appears in the near-Earth plasma sheet (around $7 R_{E}$ ), with important implications for substorm onset mechanisms. Another interesting feature also involving the transverse electric current is found when anisotropy in pressure is considered: the region of maximum current value is no longer on the equatorial plane, but instead two regions above and below the plane appear.

The 3-D force-balanced states obtained by our model yield the pressure at all locations of our computational domain, and thus our approach represents a rigorous method for obtaining a 3-D global pressure distribution. The $3-\mathrm{D}$ code results have recently been used as background configurations for computing field-line resonances in the magnetosphere [Cheng and Zaharia, 2003], and the model should also be of great use for other applications where a 3-D forcebalanced magnetospheric state is essential, such as stability analyses, plasma wave and particle simulation studies.

Acknowledgments. This work was supported by NASA grant No. W-19512 and DoE contract No. DEAC02-76-CH03073. We thank Dr. N. A. Tsyganenko for making available the computational routines for his empirical models. We acknowledge Dr. T. Mukai for the use of Geotail LEP data, and Dr. D. McCune and collaborators for the use of the NTCC PSPLINE modules.

\section{References}

Angelopoulos, et al., Characteristics of ion flow in the quiet state of the inner plasma sheet, Geophys. Res. Lett., 20, 1711, 1993.

Antonova, E. E., and N. Y. Ganyushkina, Reconstruction of large-scale azimuthal pressure gradients in the magnetosphere from field-aligned current data, Geomagn. and Aeron. (English transl.), 35, 610, 1996.

Becker, U., T. Neukirch, and K. Schindler, On the quasistatic development of thin current sheets in magnetotail-like magnetic fields, J. Geophys. Res., 106, 3811, 2001.

Birn, J., R. R. Sommer, and K. Schindler, Selfconsistent theory of the quiet magnetotail in three dimensions, J. Geophys. Res., 82, 147, 1977.

Borovsky, J. E., R. C. Elphic, H. O. Funsten, and M. F. Thomsen, The Earth's plasma sheet as a laboratory for flow turbulence in high- $\beta$ MHD, $J$. Plasma Phys., 57, 1, 1997.

Cao, F., and L. C. Lee, Plasma pressure and anisotropy inferred from the Tsyganenko magnetic-field model, Ann. Geophys., 12, 286, 1994.

Cheng, C. Z., Magnetospheric equilibrium with anisotropic pressure, J. Geophys. Res., 97, 1497, 1992.

Cheng, C. Z., Three-dimensional magnetospheric equilibrium with isotropic pressure, Geophys. Res. Lett., 22, 2401, 1995.

Cheng, C. Z., and S. Zaharia, Field line resonances in quiet and disturbed time 3-D magnetospheres, J. Geophys. Res., 128, 2003, 1001, doi:10.1029/2002JA009471. 
Cheng, C. Z., T. C. Chang, C. A. Lin, and W. H. Tsai, Magnetohydrodynamic theory of field line resonances in the magnetosphere, J. Geophys. Res., 98, 11,339-11,347, 1993.

De Michelis, P., I. A. Daglis, and G. Consolini, An average image of proton plasma pressure and of current systems in the equatorial plane derived from AMPTE/CCE-CHEM measurements, J. Geophys. Res., 104, 28,615, 1999.

Hesse, M., and J. Birn, MHD modeling of magnetotail instability for anisotropic pressure, J. Geophys. Res., 97, 10,643, 1992.

Hesse, M., and J. Birn, Three-dimensional magnetotail equilibria by numerical relaxation techniques, J. Geophys. Res., 98, 3973, 1993.

Hill, T. W., and G. H. Voigt, Limits on plasma anisotropy in a tail-like magnetic field, Geophys. Res. Lett., 19, 2441, 1992.

Hori, T., K. Maezawa, Y. Saito, and T. Mukai, Average profile of ion flow and convection electric field in the near-Earth plasma sheet, Geophys. Res. Lett., 27, 1623, 2000.

Horton, W., L. Cheung, J. Y. Kim, and T. Tajima, Self-consistent plasma pressure tensors from the Tsyganenko field models, J. Geophys. Res., 98, 17,327, 1993.

Huang, C. Y., and L. A. Frank, A statistical survey of the central plasma sheet, J. Geophys. Res., 99, 83-95, 1994.

Hudson, S. R., D. A. Monticello, A. H. Reiman, A. H. Boozer, D. J. Strickler, S. P. Hirshman, and M. C. Zarnstorff, Phys. Rev. Lett., 89, 275,003-1, 2002.

Iijima, T., and T. A. Potemra, The amplitude distribution of field-aligned currents at northern high latitudes observed by Triad, J. Geophys. Res., 81, 2165, 1976.

Kistler, L. M., E. Mobius, W. Baumjohann, G. Paschmann, and D. C. Hamilton, Pressure changes in the plasma sheet during substorm injections, J. Geophys. Res., 97, 2973, 1992.

Lui, A. T. Y., Radial transport of storm time ring current ions, J. Geophys. Res., 98, 209, 1993.

Lui, A. T. Y., and D. C. Hamilton, Radial profiles of quiet time magnetospheric parameters, J. Geophys. Res., 97, 19,325, 1992.

Lui, A. T. Y., R. W. McEntire, and S. M. Krimigis, Evolution of the ring current during two geomagnetic storms, J. Geophys. Res., 92, 7459, 1987.

Lui, A. T. Y., H. E. Spence, and D. P. Stern, Empirical modeling of the quiet time nightside magnetosphere, J. Geophys. Res., 99, 151, 1994.
Nakamura, M., G. Paschmann, W. Baumjohann, and N. Sckopke, Ion distributions and flows near the neutral sheet, J. Geophys. Res., 96, 5631, 1991.

Noetzel, A., K. Schindler, and J. Birn, On the cause of approximate pressure isotropy in the quiet nearEarth plasma sheet, J. Geophys. Res., 90, 8293, 1985.

Olson, W. P., and K. A. Pfitzer, Quantitative model of magnetospheric magnetic-field, J. Geophys. Res., 79, 3739, 1974.

Ostapenko, A. A., and Y. P. Maltsev, Relation of the magnetic field in the magnetosphere to the geomagnetic and solar wind activity, J. Geophys. Res., 102, 17,467, 1997.

Sergeev, V. A., M. Malkov, and K. Mursula, Testing the isotropic boundary algorithm method to evaluate the magnetic field configuration in the tail, $J$. Geophys. Res., 98, 7609, 1993.

Spence, H. E., and M. G. Kivelson, Contributions of the low-latitude boundary layer to the finite width magnetotail convection model, J. Geophys. Res., 98, 15,487, 1993.

Spence, H. E., M. G. Kivelson, and R. J. Walker, Static magnetic field models consistent with nearly isotropic plasma pressure, Geophys. Res. Lett., 14, 872, 1987.

Spence, H. E., M. G. Kivelson, R. J. Walker, and D. J. McComas, Magnetospheric plasma pressures in the midnight meridian: Observations from 2.5 to $35 \mathrm{R}_{\mathrm{E}}$, J. Geophys. Res., 94, 5264, 1989.

Stern, D., Geomagnetic Euler potentials, J. Geophys. Res., 72, 3995, 1967.

Stern, D., Euler potentials, Am. J. Phys., 38, 494, 1970.

Stern, D. P., The art of mapping the magnetosphere, J. Geophys. Res., 99, 17,169, 1994.

Stiles, G. S., J. E. W. Hones, S. J. Bame, and J. R. Asbridge, Plasma sheet pressure anisotropies, J. Geophys. Res., 83, 3166, 1978.

Toffoletto, A. F., B. S. Sazykin, C. R. Wolf, D. R. Spiro, E. J. Birn, and F. M. Hesse, Numerical modeling of inner magnetospheric electrodynamics, 2001.

Tsyganenko, N. A., Global quantitative models of the geomagnetic field in the cislunar magnetosphere for different disturbance levels, Planet. Space Sci., 35, $1347,1987$.

Tsyganenko, N. A., A magnetospheric field model with a warped tail current sheet, Planet. Space Sci., 37, 5, 1989. 
Tsyganenko, N. A., Quantitative models of magnetospheric magnetic field: Methods and results, Space. Sci. Rev., 54, 75, 1990.

Tsyganenko, N. A., Modeling the Earth's magnetospheric magnetic field confined within a realistic magnetopause, J. Geophys. Res., 100, 5599, 1995.

Tsyganenko, N. A., A model of the near magnetosphere with a dawn-dusk asymmetry, 2. Parameterization and fitting to observations, J. Geophys. Res., 107, 2002, 1001, doi:10.1029/2001JA000220.

Tsyganenko, N. A., and D. P. Stern, Modeling the global magnetic field of the large-scale Birkeland current systems, J. Geophys. Res., 101, 27,187, 1996.

Vasyliunas, V. M., Mathematical models of magnetospheric convection and its coupling to the ionosphere, in Particles and Fields in the Magnetosphere, ed. B. M. McCormac, D. Reidel, Hingham, MA, pp. 60-71, 1970.

Vasyliunas, V. M., Fundamentals of current description, in Magnetospheric Currents, ed. T. A. Potemra, American Geophysical Union, Washington, D. C., p. 63, 1984.

Wing, S., and P. T. Newell, Central plasma sheet ion properties as inferred from ionospheric observations, J. Geophys. Res., 103, 6785, 1998.

Wolf, R. A., The quasi-static (slow-flow) region of the magnetosphere, in Solar-Terrestrial Physics: Principles and Theoretical Foundations: based on the proceedings of the Theory Institute held at Boston College, August 9-26, 1982, pp. 303-368, Kluwer Academic, 1983.

Zaharia, S., and C. Z. Cheng, Physical limitations of empirical field models: Force balance and plasma pressure, 2002, submitted to J. Geophys. Res.

Sorin Zaharia and C. Z. Cheng, Princeton University, Plasma Physics Laboratory, P.O. Box 451, Princeton, NJ 08543. (e-mail: szaharia@pppl.gov, fcheng@pppl.gov)

K. Maezawa, Institute of Space and Astronautical Science, Yoshinodai 3-1-1, Sagamihara, Kanagawa, 229 8510, Japan. (e-mail: maezawa@stp.isas.ac.jp)

This preprint was prepared with AGU's IATEX macros v5.01. File PPPL report formatted February 3, 2003.

With the extension package ' $\mathrm{AGU}^{++}$', version 1.2 from $1995 / 01 / 12$ 


\section{External Distribution}

Plasma Research Laboratory, Australian National University, Australia

Professor I.R. Jones, Flinders University, Australia

Professor João Canalle, Instituto de Fisica DEQ/IF - UERJ, Brazil

Mr. Gerson O. Ludwig, Instituto Nacional de Pesquisas, Brazil

Dr. P.H. Sakanaka, Instituto Fisica, Brazil

The Librarian, Culham Laboratory, England

Mrs. S.A. Hutchinson, JET Library, England

Professor M.N. Bussac, Ecole Polytechnique, France

Librarian, Max-Planck-Institut für Plasmaphysik, Germany

Jolan Moldvai, Reports Library, MTA KFKI-ATKI, Hungary

Dr. P. Kaw, Institute for Plasma Research, India

Ms. P.J. Pathak, Librarian, Insitute for Plasma Research, India

Ms. Clelia De Palo, Associazione EURATOM-ENEA, Italy

Dr. G. Grosso, Instituto di Fisica del Plasma, Italy

Librarian, Naka Fusion Research Establishment, JAERI, Japan

Library, Plasma Physics Laboratory, Kyoto University, Japan

Research Information Center, National Institute for Fusion Science, Japan

Dr. O. Mitarai, Kyushu Tokai University, Japan

Library, Academia Sinica, Institute of Plasma Physics, People's Republic of China

Shih-Tung Tsai, Institute of Physics, Chinese Academy of Sciences, People's Republic of China

Dr. S. Mirnov, TRINITI, Troitsk, Russian Federation, Russia

Dr. V.S. Strelkov, Kurchatov Institute, Russian Federation, Russia

Professor Peter Lukac, Katedra Fyziky Plazmy MFF UK, Mlynska dolina F-2, Komenskeho Univerzita, SK-842 15 Bratislava, Slovakia

Dr. G.S. Lee, Korea Basic Science Institute, South Korea

Institute for Plasma Research, University of Maryland, USA

Librarian, Fusion Energy Division, Oak Ridge National Laboratory, USA

Librarian, Institute of Fusion Studies, University of Texas, USA

Librarian, Magnetic Fusion Program, Lawrence Livermore National Laboratory, USA

Library, General Atomics, USA

Plasma Physics Group, Fusion Energy Research Program, University of California at San Diego, USA

Plasma Physics Library, Columbia University, USA

Alkesh Punjabi, Center for Fusion Research and Training, Hampton University, USA

Dr. W.M. Stacey, Fusion Research Center, Georgia Institute of Technology, USA

Dr. John Willis, U.S. Department of Energy, Office of Fusion Energy Sciences, USA

Mr. Paul H. Wright, Indianapolis, Indiana, USA 
The Princeton Plasma Physics Laboratory is operated by Princeton University under contract with the U.S. Department of Energy.

\author{
Information Services \\ Princeton Plasma Physics Laboratory \\ P.O. Box 451 \\ Princeton, NJ 08543
}

Phone: 609-243-2750

Fax: 609-243-2751

e-mail: pppl_info@pppl.gov

Internet Address: http://www.pppl.gov 